\title{
2-Deoxyribose as a Rich Source of Chiral 5-Carbon Building Blocks ${ }^{\S}$
}

\author{
Dengjin Wang* and William A. Nugent* \\ Bristol-Myers Squibb Company, Process Research and Development, \\ P. O. Box 4000, Princeton NJ 08543 \\ dengjin.wang@bms.com
}

General Experimental.................................2

Additional Synthetic Details

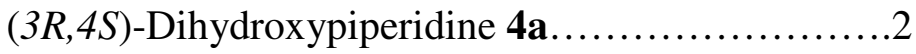

Iodolactone 11...................................3

Propargylic Sulfinamide 21...........................4

Propargylic Sulfinamide 22......................5

References...........................................5

Additional Characterization of Products

2D NMR Analysis for 20.......................6

Chiral GC Analysis for $5 \ldots \ldots \ldots \ldots \ldots \ldots \ldots \ldots \ldots . \ldots . \ldots$

DSC Analysis of Lactone 10.....................10

DSC Analysis of Propargylic Sulfinamide 21.........11

${ }^{1} \mathrm{H}$ and ${ }^{13} \mathrm{C}$ NMR Spectra

Carboxylic Acid 2..............................12

Aldehyde 3...................................... 14

Dihydroxypiperidine 4.............................. 16

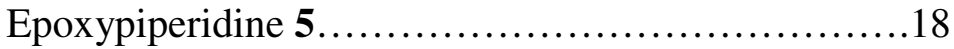

Lactone 10...................................20

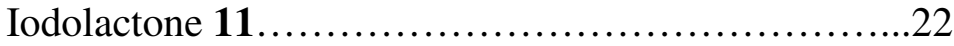

Weinreb Amide 12...............................24

Iodo Acetal 15.....................................26

Chloro Acetate 20................................28

Propargylic Sulfinamide 21.........................30

Propargylic Sulfinamide 22.........................32 
General. NMR spectra were determined at $25^{\circ} \mathrm{C}$ in solvent $\mathrm{CDCl}_{3}$ unless otherwise noted at a field strength of $400 \mathrm{MHz}\left({ }^{1} \mathrm{H}\right.$ spectra $)$ or $100 \mathrm{MHz}\left({ }^{13} \mathrm{C}\right.$ spectra). Anhydrous solvents tetrahydrofuran, dichloromethane, and dimethylformamide as well as all synthetic reagents were commercial materials used as received. Flash chromatography was performed on 220-400 mesh silica (E. Merck) following the standard procedure of Still. ${ }^{1}$ Reactions were run under an atmosphere of dry nitrogen. Melting points were determined by differential scanning calorimetry (DSC) and are reported as peak minima.

\section{Additional Synthetic Details}

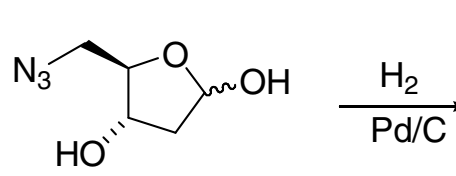

17

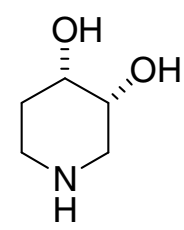

$4 a$

(3R,4S)-Dihydroxypiperidine, 4a. Removal of the last traces of ethanol from the Nunprotected piperidine $\mathbf{4 a}$ is difficult and the freebase is less stable during storage under air than its Boc-protected analogue 4 . If storage of $\mathbf{4 a}$ is required, we recommend its conversion to its hydrochloride with anhydrous $\mathrm{HCl}$. The hydrochloride is a well behaved (but hygroscopic) solid which can be freebased as needed using triethylamine or $\mathrm{NaOH}$. Nevertheless, compound $4 \mathbf{a}$ was prepared in order to compare its spectroscopic properties with those reported by Wong and coworkers. ${ }^{2}$

A Fischer-Porter tube was charged with a solution of azide $\mathbf{1 7}(1.10 \mathrm{~g}, 6.9 \mathrm{mmol})$ in anhydrous ethanol $(25 \mathrm{~mL})$. After addition of $5 \%$ palladium on carbon catalyst $(0.30$ g), the system was flushed with nitrogen and pressured to 60 psi. Stirring was continued $48 \mathrm{~h}$ at room temperature, repressuring to $60 \mathrm{psi}$ as needed. (Note that during the early 
stages of the reaction, there is minimal pressure drop since one mol of nitrogen is generated for each mol of hydrogen consumed.) The solution was then filtered and the solvent was removed at reduced pressure. In order to provide an ethanol-free NMR spectrum, a pipette of methanol- $\mathrm{d}_{4}$ was added and then distilled at reduced pressure. The final weight of $\mathbf{4 a}$ was $0.81 \mathrm{~g}$ of nearly colorless oil. The NMR spectra were essentially identical to those reported by Wong and coworkers ${ }^{2}$ for material prepared by their chemoenzymatic route. ${ }^{1} \mathrm{H}$ NMR (400 MHz, $\left.\mathrm{D}_{2} \mathrm{O}\right) \delta$ : 1.55 (2H, overlapping m), 2.43 (1H, ddd, $J=13.3,7.9,4.2), 2.55(1 \mathrm{H}, \mathrm{dd}, J=13.4,2.5), 2.72(2 \mathrm{H}$, overlapping $\mathrm{m}), 3.64$ $(1 \mathrm{H}, \mathrm{dt}, J=6.1,2.8), 3.73(1 \mathrm{H}, \mathrm{m}) .{ }^{13} \mathrm{C} \mathrm{NMR}\left(100 \mathrm{MHz}, \mathrm{D}_{2} \mathrm{O}\right) \delta: 68.9,68.4,47.7,41.6$, 29.6. Because of its tendency to retain solvent, satisfactory elemental analysis of $\mathbf{4 a}$ was not obtained.

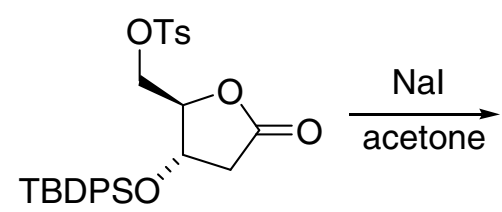

10

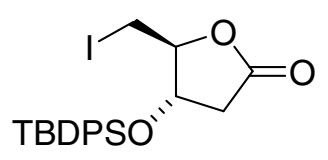

11

\section{2,5-Dideoxy-1-O-methyl-3-O-tert-butyldiphenylsilyl-5-iodo-D-ribonolactone, $11 . \quad \mathrm{A}$}

mixture of tosylate $10(2.60 \mathrm{~g}, 5.00 \mathrm{mmol})$ and sodium iodide $(1.50 \mathrm{~g}, 20.0 \mathrm{mmol})$ in acetone $(20 \mathrm{~mL})$ was heated overnight at reflux. After cooling, the mixture was diluted with ethyl acetate $(50 \mathrm{~mL})$ and filtered through a short bed of Celite. The resulting solution was washed with water $(50 \mathrm{~mL})$ and then with $5 \%$ aqueous sodium thiosulfate solution $(25 \mathrm{~mL})$. The organic phase was dried $\left(\mathrm{MgSO}_{4}\right)$ and the solvent was distilled at reduced pressure to afford $11(2.39 \mathrm{~g}, 100 \%)$ as a nearly colorless oil. ${ }^{1} \mathrm{H}$ NMR (400 $\left.\mathrm{MHz}_{\mathrm{CDCl}}\right)$ $)$ : $7.57(4 \mathrm{H}, \mathrm{m})$, 7.46-7.32 (6H, overlapping m), $4.26(1 \mathrm{H}, \mathrm{m}), 2.95(1 \mathrm{H}$, $\mathrm{dd}, J=11.0,5.3), 2.73-2.62(2 \mathrm{H}$, overlapping $\mathrm{m}), 2.46(1 \mathrm{H}, \mathrm{dd}, J=18.0,3.5), 1.01(9 \mathrm{H}$, 
s). ${ }^{13} \mathrm{C} \mathrm{NMR}\left(100 \mathrm{MHz}, \mathrm{CDCl}_{3}\right) \delta: 173.8,135.7,135.6,132.4,128.1,128.0,85.5,72.9$,

38.1, 26.7, 18.9, 3.8. Anal. Calcd for $\mathrm{C}_{21} \mathrm{H}_{25} \mathrm{IO}_{3} \mathrm{Si}$ : C, 52.50; H, 5.25. Found: C, 52.27; H, 5.00 .
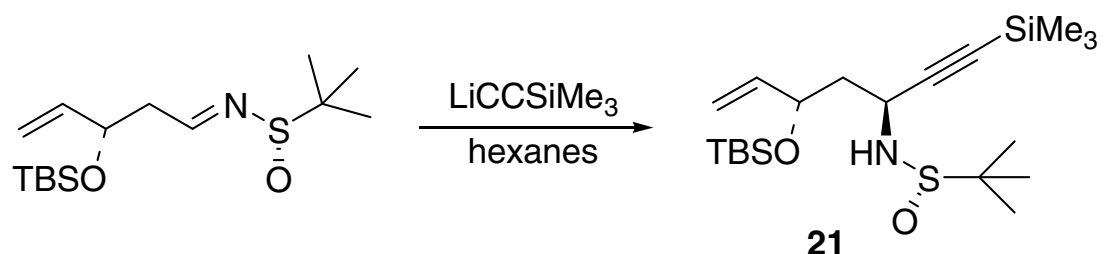

\section{$\left(S_{\mathrm{S}}, 3 S, 5 S\right)$-3-tert-butyldimethylsilyloxy-5-tert-butanesulfinamido-7-trimethylsilyl-1-}

en-6-yne, 21. The sulfinyl imine starting material was first prepared from $\mathbf{3}$ and $\left(S_{\mathrm{S}}\right)$-tertbutanesulfinamide following the general procedure of Ellman. ${ }^{3}{ }^{1} \mathrm{H}$ NMR $(400 \mathrm{MHz}$, $\left.\mathrm{CDCl}_{3}\right): \delta 8.04(1 \mathrm{H}, \mathrm{t}, \mathrm{J}=5.1 \mathrm{~Hz}), 5.81(1 \mathrm{H}, \mathrm{m}), 5.21(1 \mathrm{H}, \mathrm{dt}, J=17.1,1.3), 5.05(1 \mathrm{H}$, dt, $J=10.1,1.3), 4.46(1 \mathrm{H}, \mathrm{q}, J=5.7), 2.66(2 \mathrm{H}, \mathrm{m}), 1.14(9 \mathrm{H}, \mathrm{s}), 0.84(9 \mathrm{H}, \mathrm{s}), 0.02(3 \mathrm{H}$, s), $0.00(3 \mathrm{H}, \mathrm{s}) .{ }^{13} \mathrm{C} \mathrm{NMR}\left(100 \mathrm{MHz}, \mathrm{CDCl}_{3}\right): \delta 167.4,140.0,114.9,71.1,56.4,44.2$, 25.7, 22.6, 18.0, -4.6, -5.0.

Following the general procedure ${ }^{4}$ of Hou and coworkers, a solution of trimethylsilylacetylene $(1.00 \mathrm{~mL}, 7.25 \mathrm{mmol}, 2.3$ equiv) in hexanes $(50 \mathrm{~mL})$ was cooled to $-78{ }^{\circ} \mathrm{C}$. A $1.0 \mathrm{M}$ solution of lithium bis(trimethylsilyl)amide in hexanes $(7.25 \mathrm{~mL}$, $7.25 \mathrm{mmol}, 2.3$ equiv) was added. After an additional $10 \mathrm{~min}$ at $-78^{\circ} \mathrm{C}$, the sulfinyl imine $(1.00 \mathrm{~g}, 3.15 \mathrm{mmol})$ was added as a solution in $5 \mathrm{ml}$ THF $(5 \mathrm{~mL})$. The dry ice bath was removed and the solution was warmed to room temperature. Saturated $\mathrm{NH}_{4} \mathrm{Cl}(10 \mathrm{~mL})$ was added with stirring. The organic phase was separated and washed with water $(3 \times 20$ $\mathrm{mL})$ and brine $(2 \times 20 \mathrm{~mL})$. The solvent was distilled at reduced pressure and the residue was purified by flash chromatography in 70:30 hexanes/ethyl acetate to afford 21 (1.08 g, 
$82 \%)$ as a white solid, m.p. $65{ }^{\circ} \mathrm{C} .{ }^{1} \mathrm{H}$ NMR $\left(400 \mathrm{MHz}, \mathrm{CDCl}_{3}\right): \delta 5.66(1 \mathrm{H}, \mathrm{m}), 5.09$ $(1 \mathrm{H}, \mathrm{d}, \mathrm{J}=17), 4.99(1 \mathrm{H}, \mathrm{d}, \mathrm{J}=11), 4.31(1 \mathrm{H}, \mathrm{q}, \mathrm{J}=6.6), 4.04(1 \mathrm{H}, \mathrm{q}, \mathrm{J}=6.6), 3.61(1 \mathrm{H}$, $\mathrm{d}, \mathrm{J}=6.6), 1.81(2 \mathrm{H}, \mathrm{m}), 1.12(9 \mathrm{H}, \mathrm{s}), 0.81(9 \mathrm{H}, \mathrm{s}), 0.08(9 \mathrm{H}, \mathrm{s}), 0.00(3 \mathrm{H}, \mathrm{s}),-0.04(3 \mathrm{H}$, s). ${ }^{13} \mathrm{C}$ NMR $\left(100 \mathrm{MHz}, \mathrm{CDCl}_{3}\right): \delta 140.7,115.4,105.6,89.8,72.0,56.3,45.1,44.8$, 26.1, 22.8, 18.3, 0.0, -3.95, -4.42. Anal. Calcd for $\mathrm{C}_{20} \mathrm{H}_{41} \mathrm{NO}_{2} \mathrm{SSi}_{2}$ : C, 57.78; H, 9.94; N, 3.37. Found: C, 57.63; H, 9.96; N, 3.40.

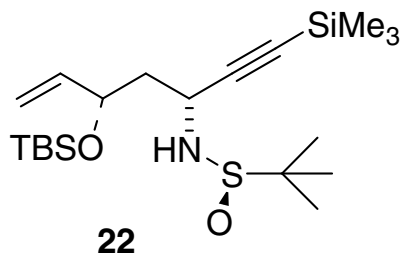

$\left(R_{\mathrm{S}}, 3 S, 5 R\right)$-3-tert-butyldimethylsilyloxy-5-tert-butanesulfinamido-7-trimethylsilyl-1en-6-yne, 22. Compound was prepared in analogous fashion to 21, starting with $\left(R_{\mathrm{S}}\right)$ tert-butanesulfinamide. ${ }^{1} \mathrm{H}$ NMR $\left(400 \mathrm{MHz}, \mathrm{CDCl}_{3}\right): \delta 5.72(1 \mathrm{H}, \mathrm{m}), 5.08(1 \mathrm{H}, \mathrm{d}, \mathrm{J}=$ 17.1), $5.02(1 \mathrm{H}, \mathrm{d}, \mathrm{J}=10.6), 4.25(1 \mathrm{H}, \mathrm{br} \mathrm{m}), 4.12(1 \mathrm{H}, \mathrm{dt}, \mathrm{J}=10.1,5.7), 3.24(1 \mathrm{H}, \mathrm{d}, \mathrm{J}=$ 5.7), $1.83(1 \mathrm{H}, \mathrm{m}), 1.64(1 \mathrm{H}, \mathrm{m}), 1.11(9 \mathrm{H}, \mathrm{s}), 0.80(9 \mathrm{H}, \mathrm{s}), 0.08(9 \mathrm{H}, \mathrm{s}), 0.00(3 \mathrm{H}, \mathrm{s}),-$ $0.05(3 \mathrm{H}, \mathrm{s}) .{ }^{13} \mathrm{C}$ NMR $\left(100 \mathrm{MHz}, \mathrm{CDCl}_{3}\right): \delta 141.5,114.7,105.3,90.4,71.5,56.1,46.0$, 26.1, 22.7, 18.3, 0.0. -3.9, -4.7.

\section{References}

1. Still, W. C.; Kahn, M.; Mitra, A. J. Org. Chem. 1978, 43, 2923.

2. Kajimoto, T.; Chen, L.; Liu, K. K.-C.; Wong, C.-H. J. Am. Chem. Soc. 1991, 113, 6678.

3. Tang, T. P.; Volkman, S. K.; Ellman, J. A. J. Org. Chem. 2001, 66, 8772.

4. Ding, C.H.; Chen, D.-D.; Luo, Z.-B.; Dai, L.-X.; Hou, X.-L. Synlett 2006, 1272. 


\section{Additional Characterization Studies}

2-D NMR Analysis for Chloro Acetate 20. The room temperature NMR of 20 was complicated by the existence of carbamate rotamers (see ${ }^{1} \mathrm{H}$ and ${ }^{13} \mathrm{C}$ spectra on pages $\mathrm{S}$ 28 and S-29 respectively). Consequently a gradient COSY study was carried out in DMSO at $100{ }^{\circ} \mathrm{C}$ in order to establish the regiochemistry of 20. Under these conditions it could be shown that the proton on the chlorine-bearing carbon at $\delta 4.23$ was strongly coupled to the $\mathrm{C} 5$ methylene and not to either $\mathrm{NCH}_{2}$ resonance. In contrast, the proton on the acetate-bearing carbon at $\delta 4.66$ was not strongly couple to the $\mathrm{C} 5$ methylene but was strongly coupled to one of the $\mathrm{NCH}_{2}$ resonances. The confirms the assignment of the structure as 3-acetato-4-chloropiperidine as proposed. The gradient COSY spectrum is shown on page $\mathrm{S}-7$. 


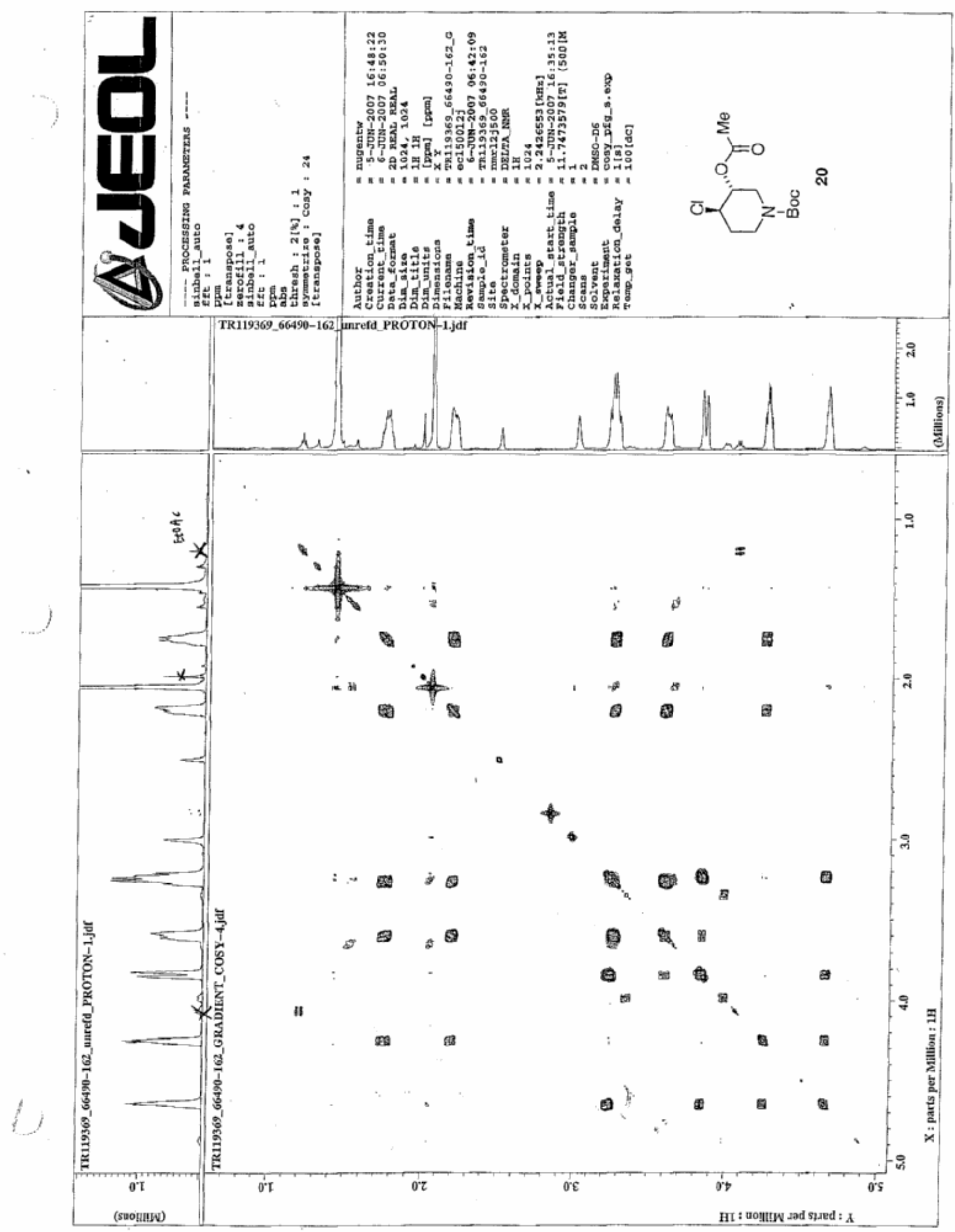


Jata File C: \HPCHBM $\backslash B 50 L 225 B \backslash D A T A \backslash Z B 5509 A \backslash 005 F 0501 . D$

b-DEX-5m, 30x0.25, $0.25 \mathrm{u}, 40 \mathrm{~cm} / \mathrm{sec}, 110 \mathrm{c} \mathrm{C}(30 \mathrm{~min})$ to 220

'C $920^{\circ} \mathrm{C} / \mathrm{min}$, hold $5 \mathrm{mins}$
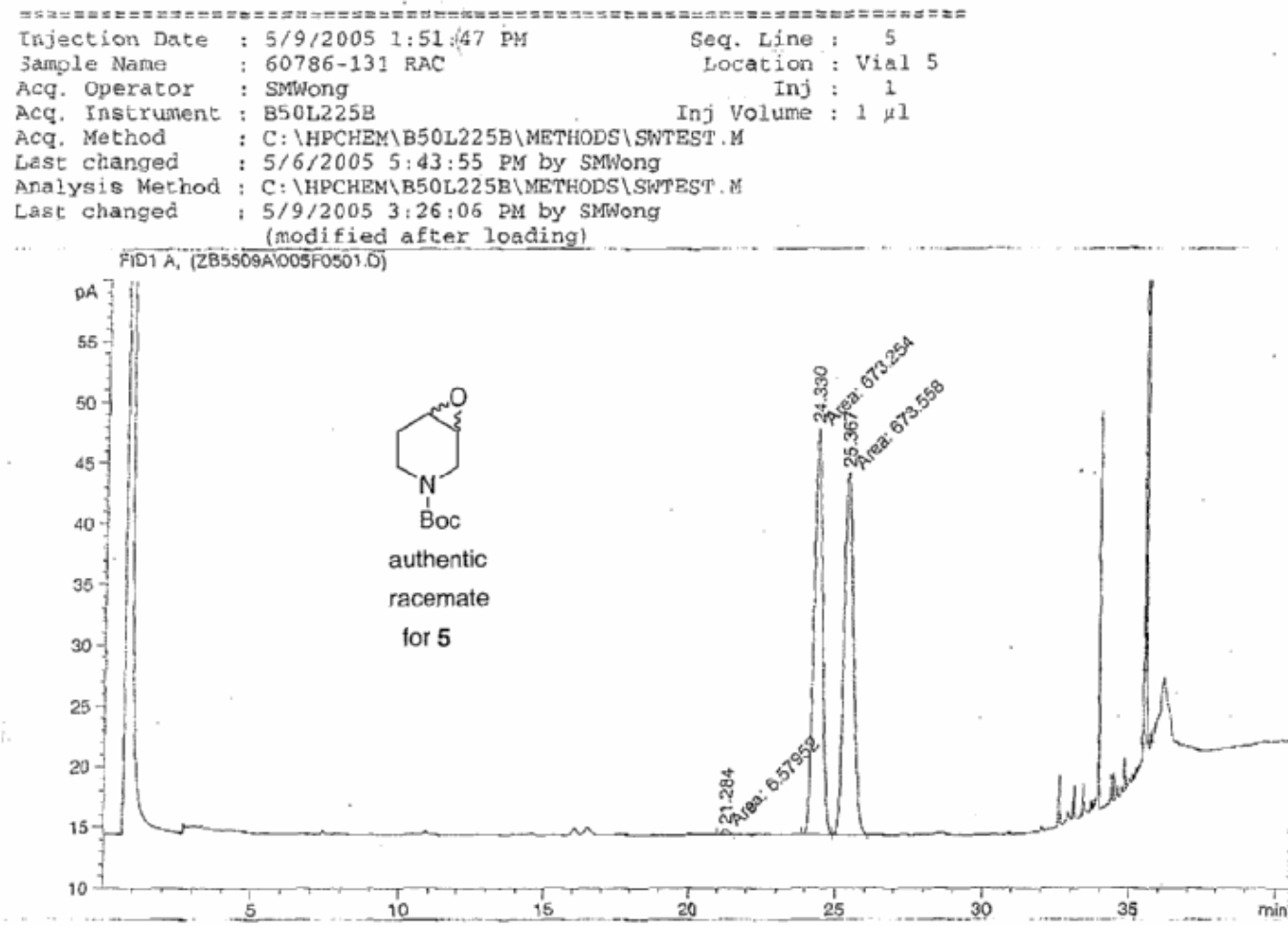

Area Percent Report

\begin{tabular}{|c|c|c|}
\hline Sorted By & : & Signal \\
\hline Multiplier & : & 1.0000 \\
\hline Dilution & $:$ & 1.0000 \\
\hline
\end{tabular}

Dilution

Use Multiplier \& Dilution Factor with ISTDs

Signal 1: FID2 A,

\begin{tabular}{|c|c|c|c|c|c|c|}
\hline eak & $\begin{array}{l}\text { RetTime } \\
\text { [min] }\end{array}$ & Type & $\begin{array}{l}\text { Width } \\
\text { [min] }\end{array}$ & $\begin{array}{r}\text { Area } \\
{\left[p A^{*} s\right]}\end{array}$ & $\begin{array}{l}\text { Height } \\
\text { [pA] }\end{array}$ & $\begin{array}{c}\text { Area } \\
8\end{array}$ \\
\hline 1 & 21.284 & $\mathrm{M}$ & $0.27-2$ & 6.57952 & $4.03555 e-1$ & 0.48615 \\
\hline 2 & 24.330 & MM & & 673.25372 & 33. 49583 & 49.74567 \\
\hline 3 & 25.367 & MM & 0. & 673.55847 & 29.80087 & 49.76818 \\
\hline
\end{tabular}

Totals : $\quad 1353.39171 \quad 63.70025$

Results obtained with enhanced integrator!

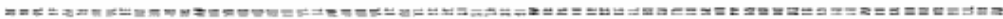

$* * *$ End of Report *** 


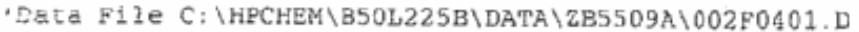
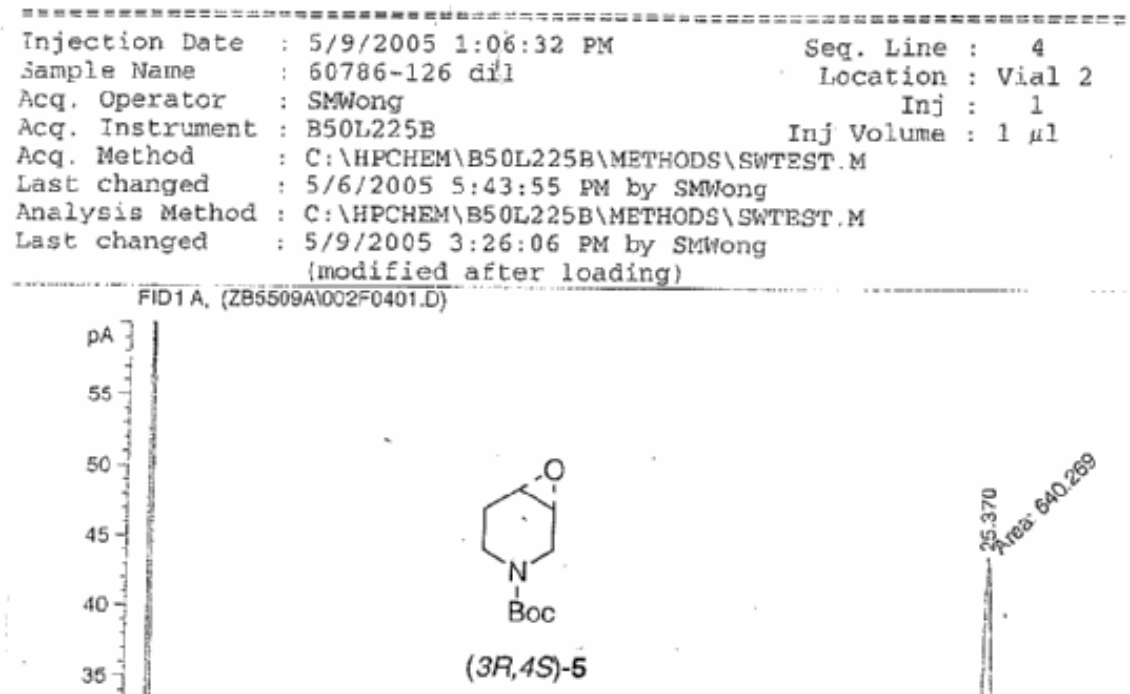

30

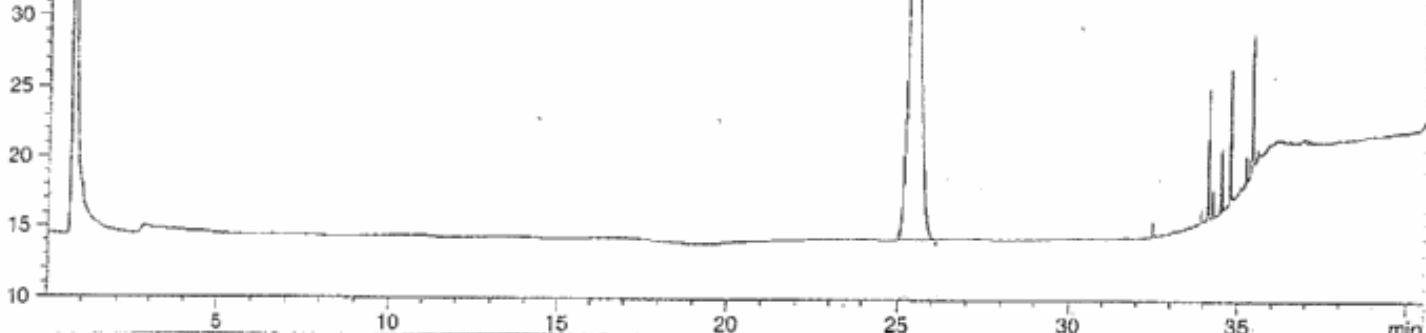

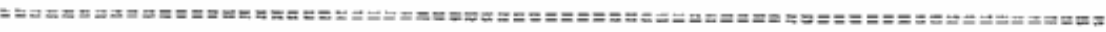
Area Percent Report

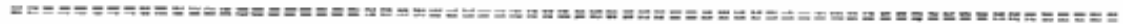

Sorted By

Multiplier

Dilution

1.0000

Use Multiplier \& Dilution Factor with ISTDs

Signal 1: FID1. A,

\begin{tabular}{|c|c|c|c|c|c|}
\hline $\begin{array}{c}\text { Peak } \\
\sharp\end{array}$ & $\begin{array}{l}\text { Retrime Type } \\
\text { [min] }\end{array}$ & $\begin{array}{l}\text { Width } \\
\text { [min] }\end{array}$ & $\begin{array}{r}\text { Area } \\
{\left[p A^{*} s\right]}\end{array}$ & $\begin{array}{l}\text { Height } \\
\text { [pA] }\end{array}$ & $\begin{array}{c}\text { Area } \\
\text { \& }\end{array}$ \\
\hline 1 & $25.370 \mathrm{Mm}$ & 0.3630 & 640.26935 & 29.39548 & $1.000 \mathrm{e} 2$ \\
\hline & & & 640 & 9548 & \\
\hline
\end{tabular}

Results obtained with enhanced integrator! 
S-10

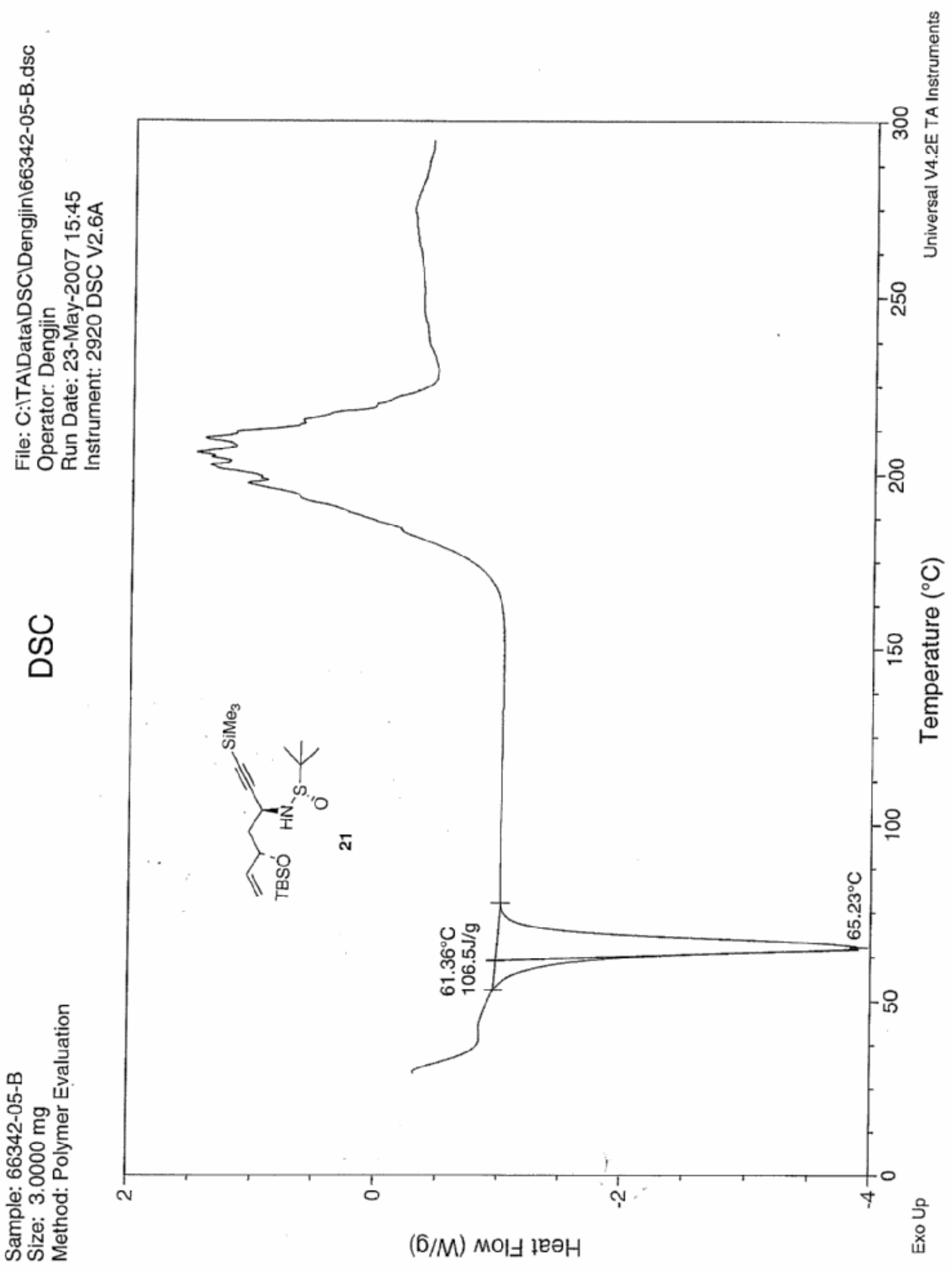


S-11

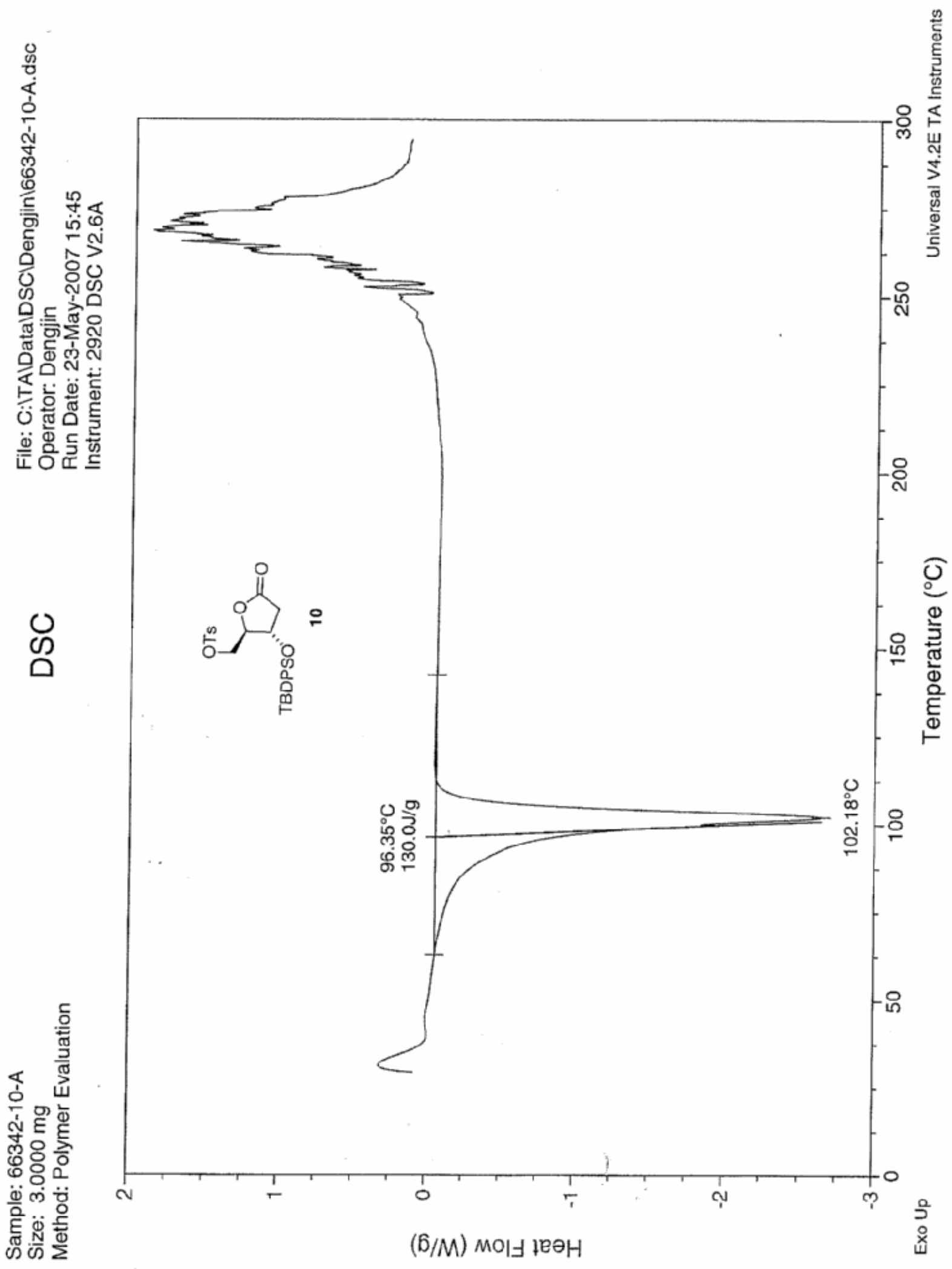




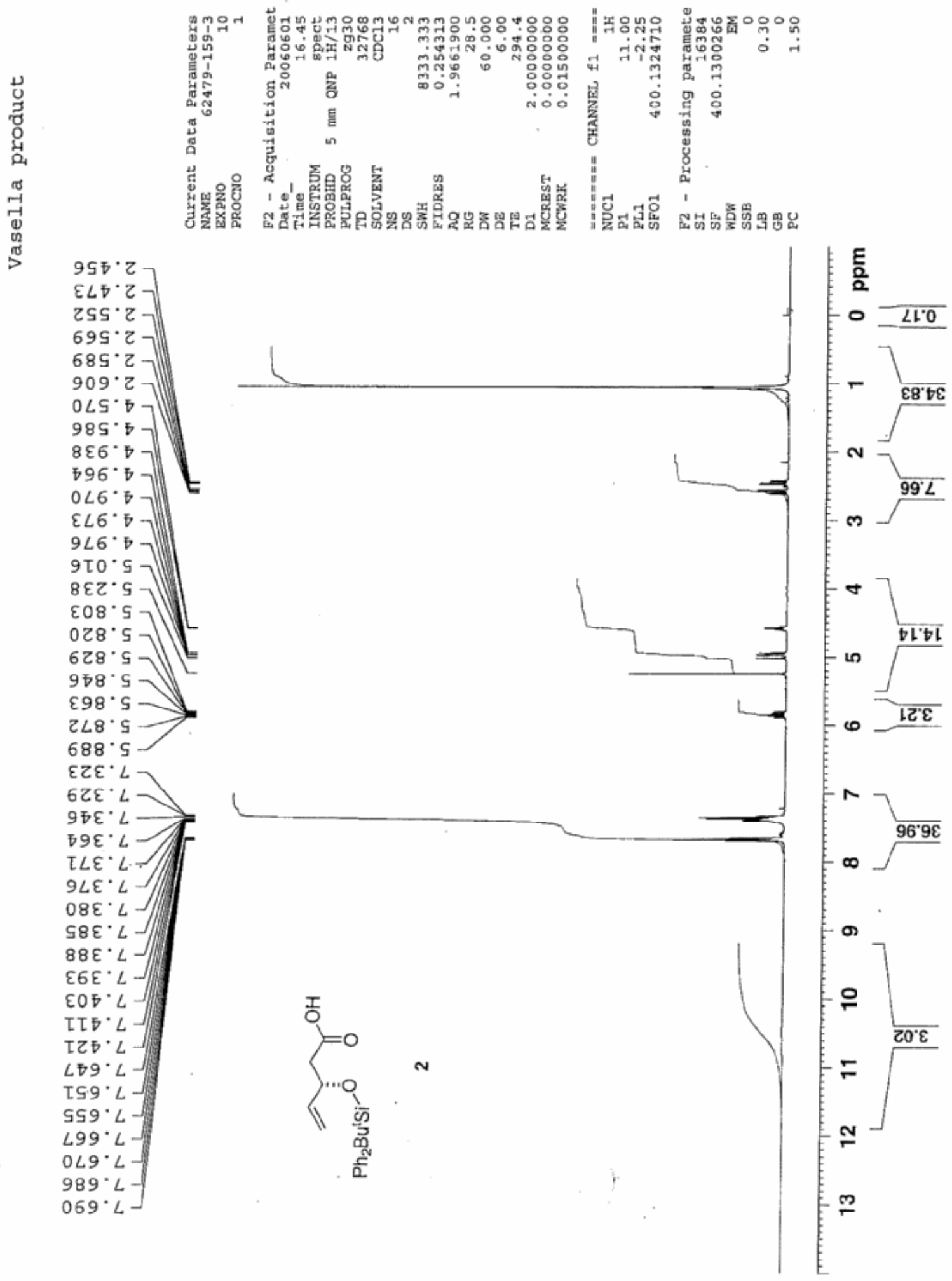



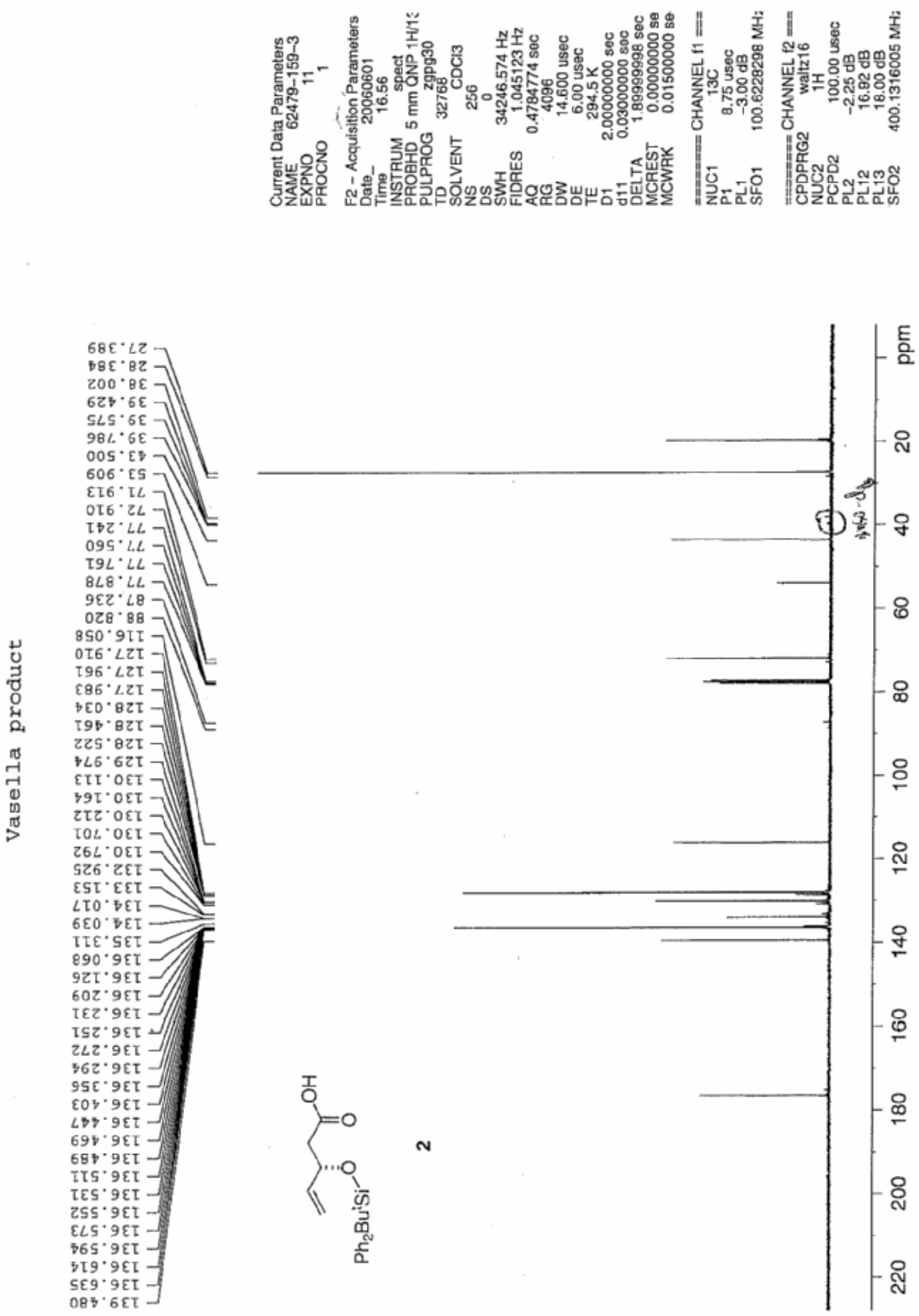
S-14

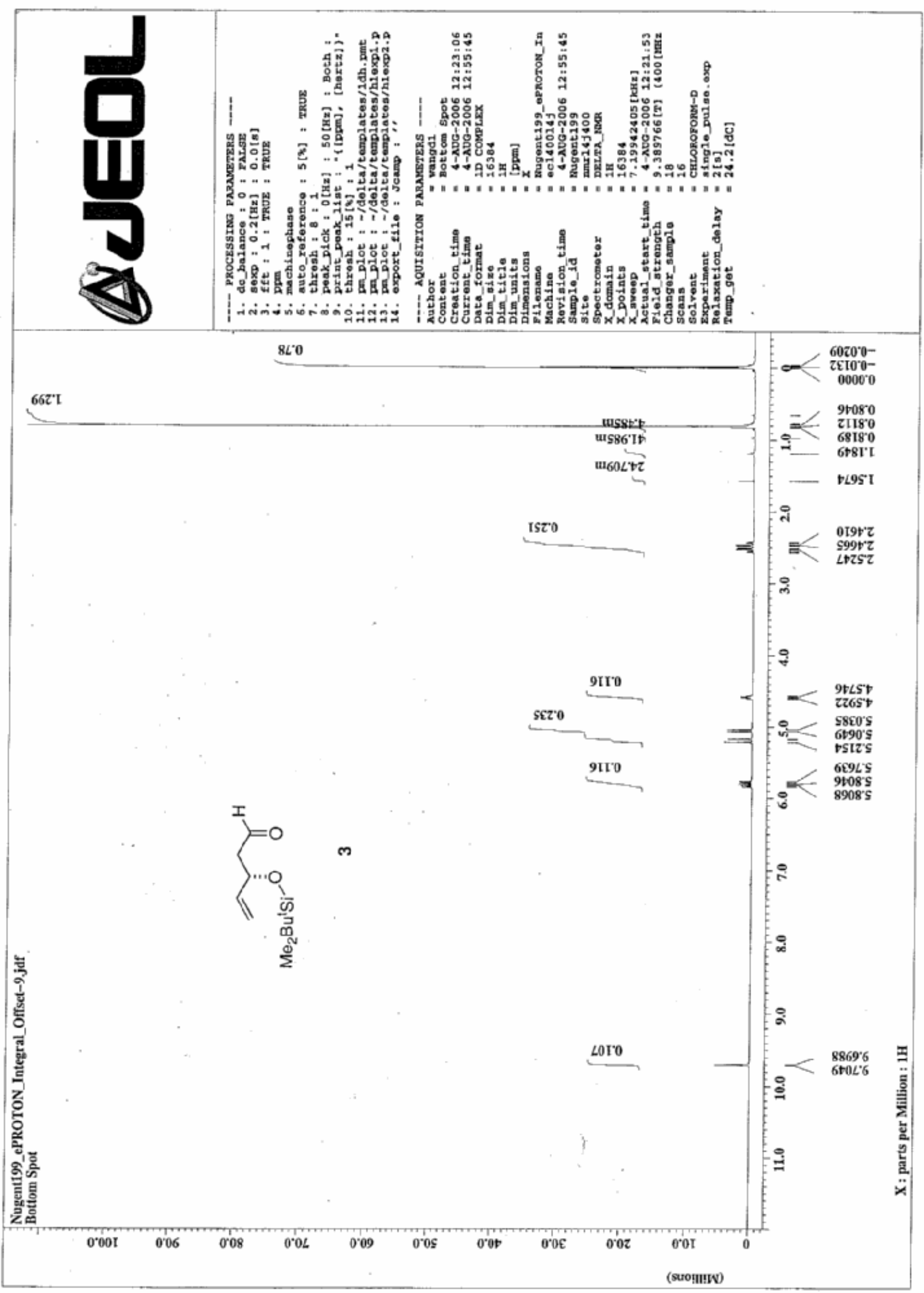


S-15

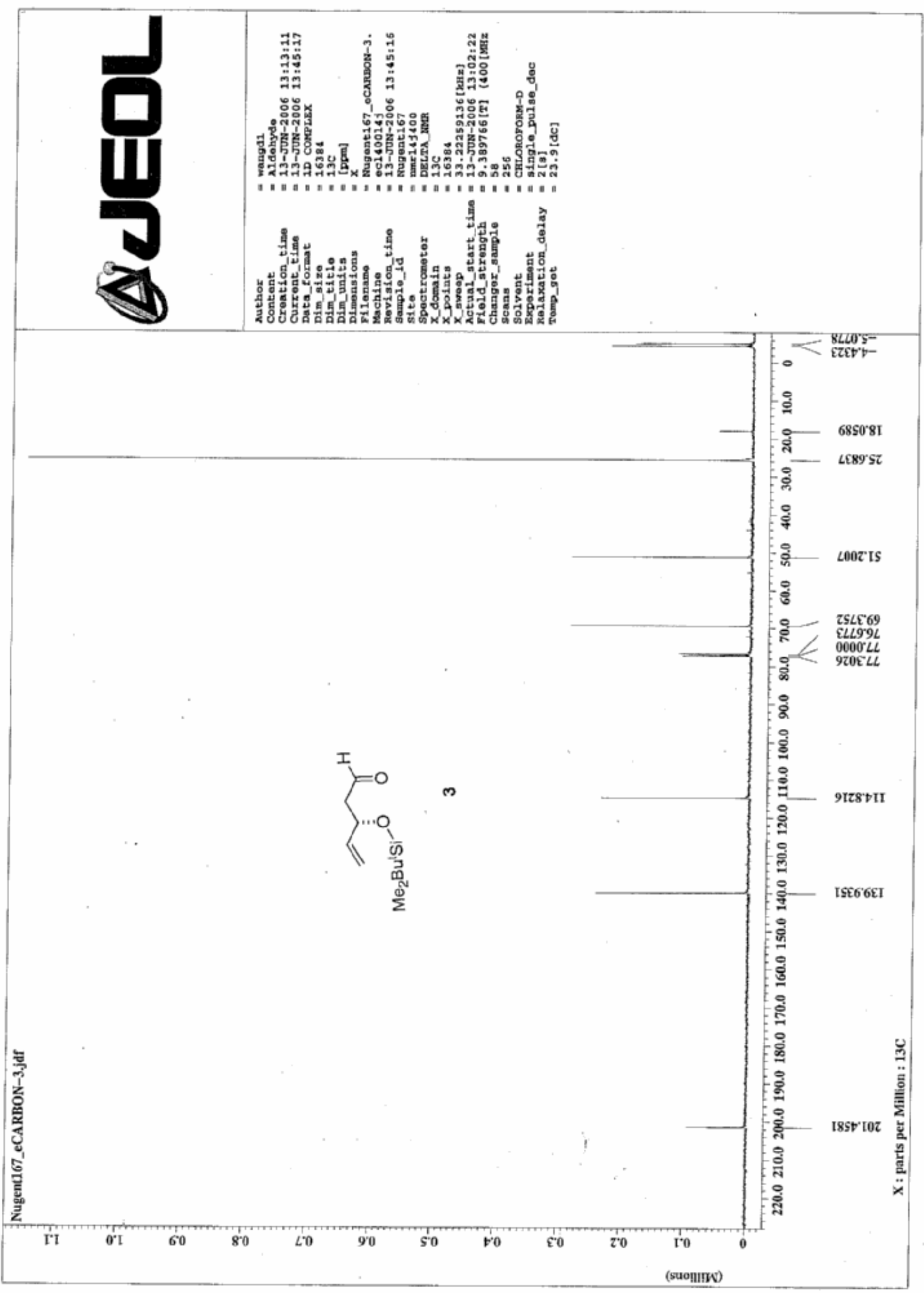


S-16
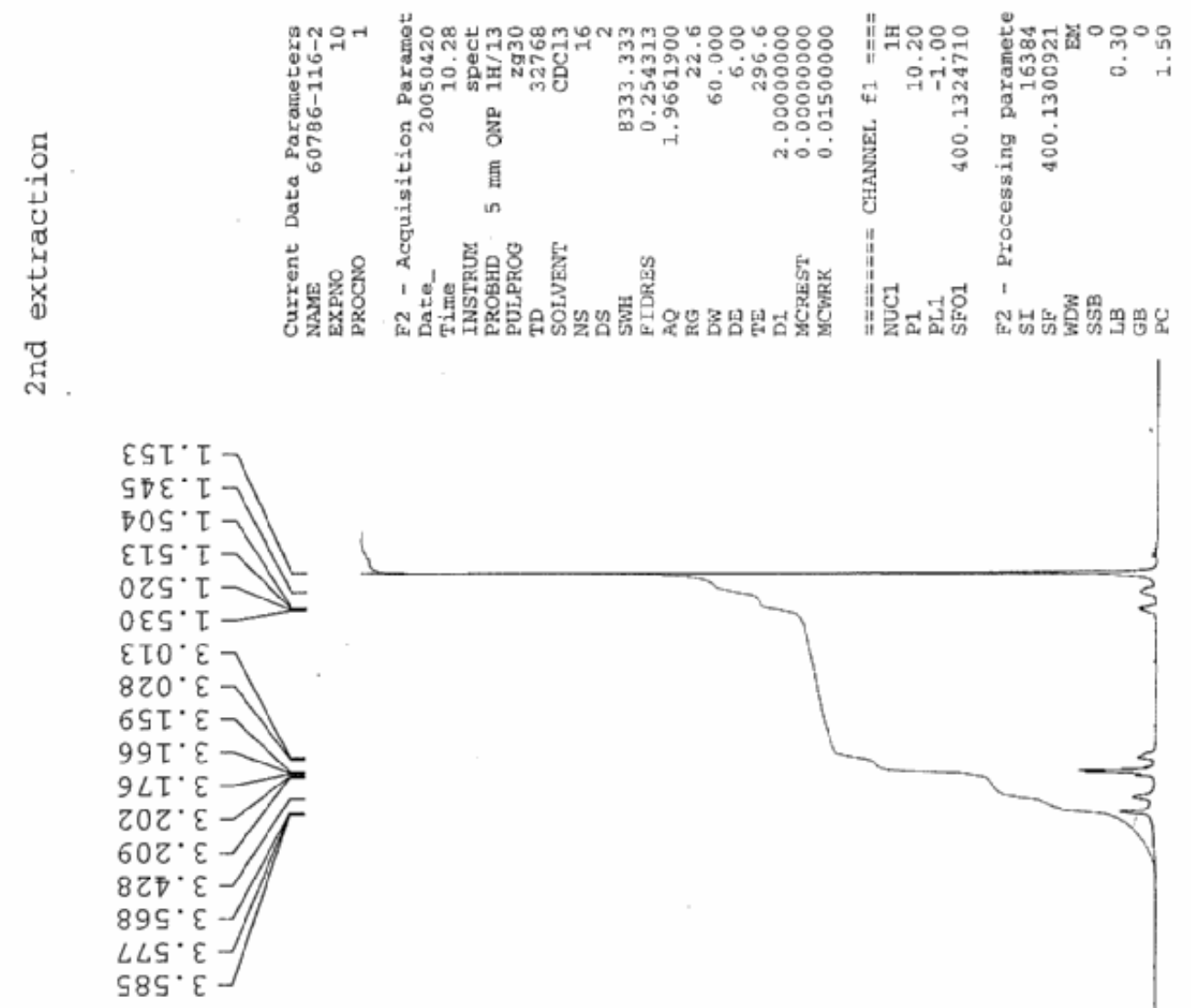

$\varepsilon \$ 0^{\circ} L-$

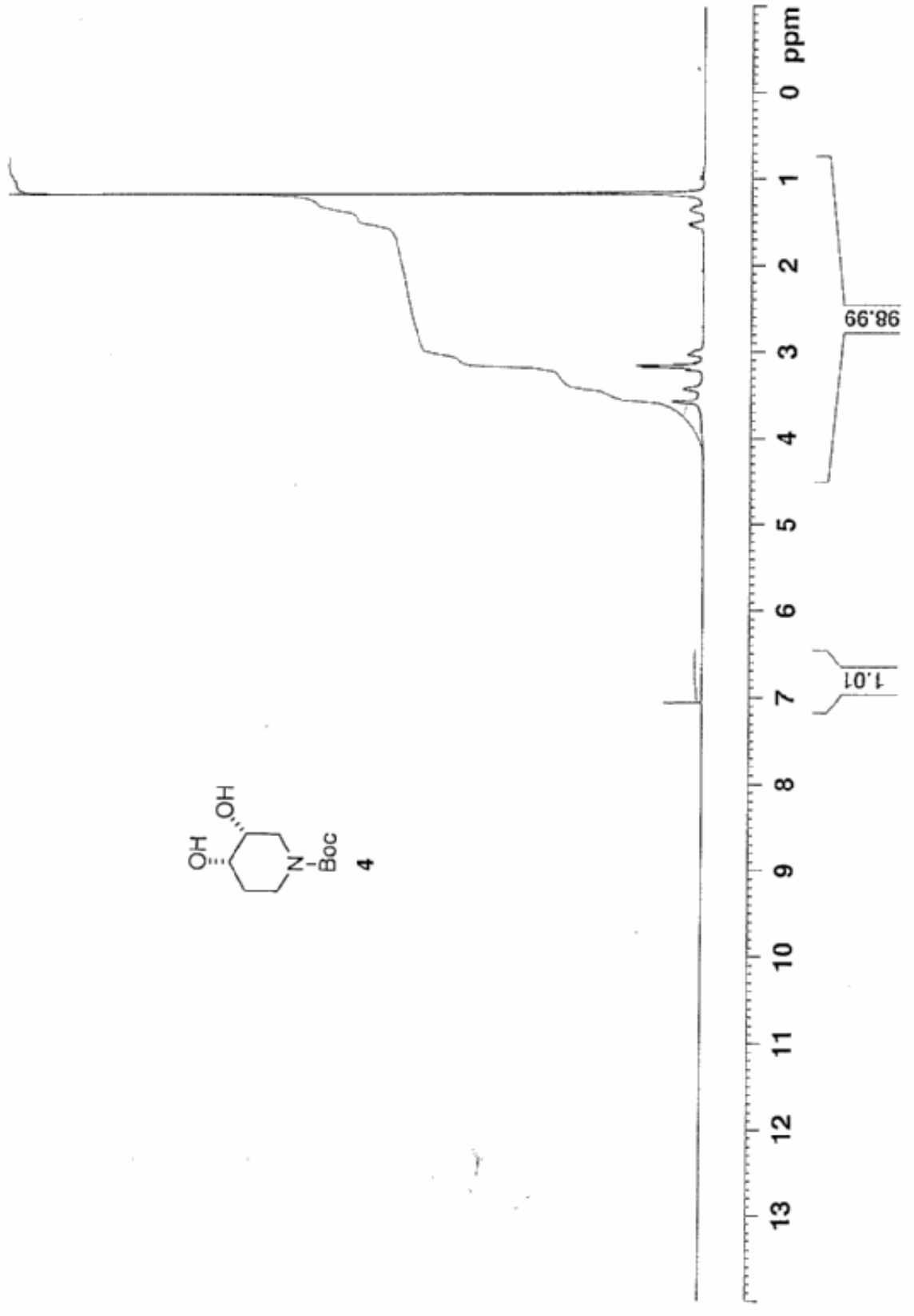



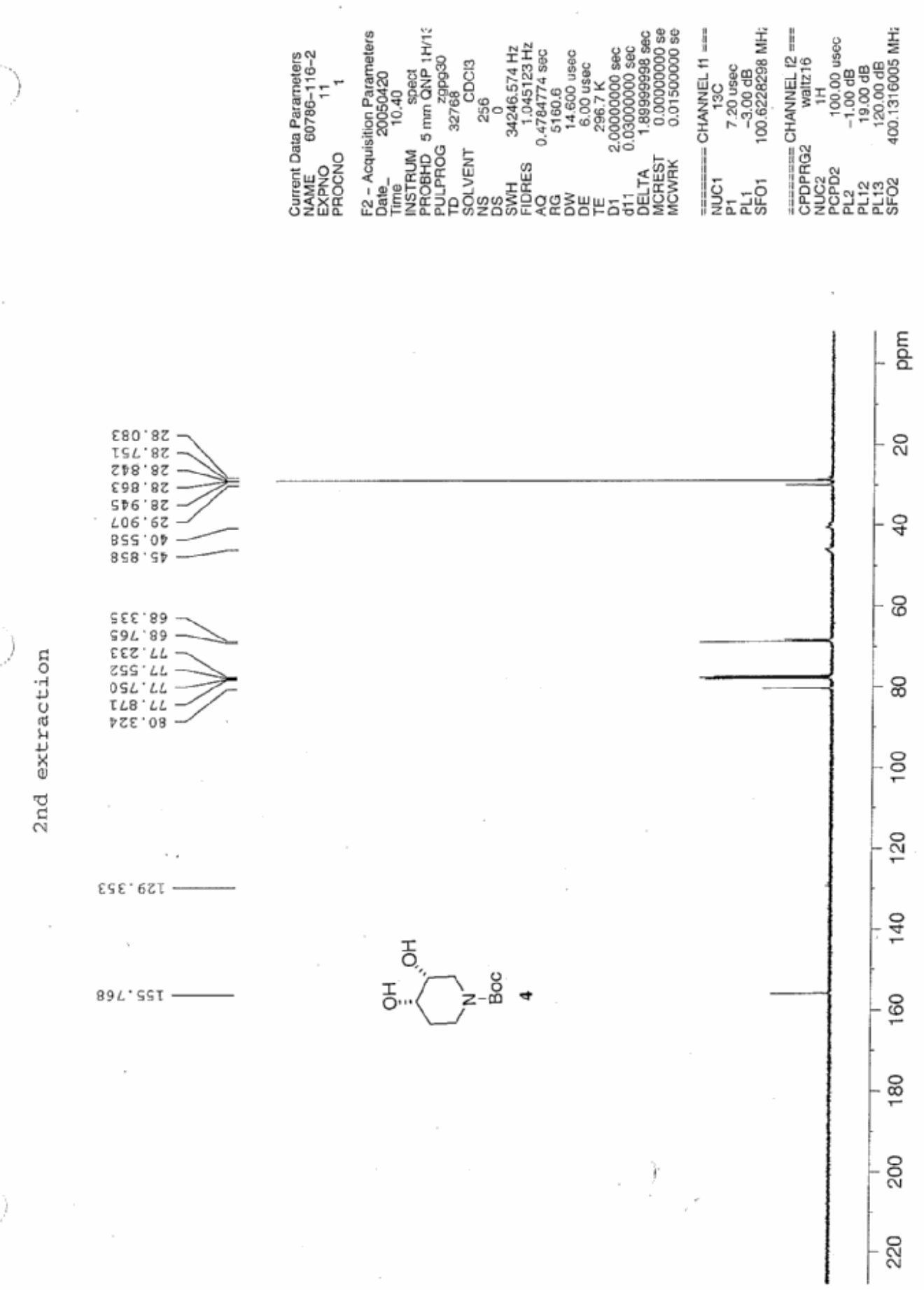
S-18

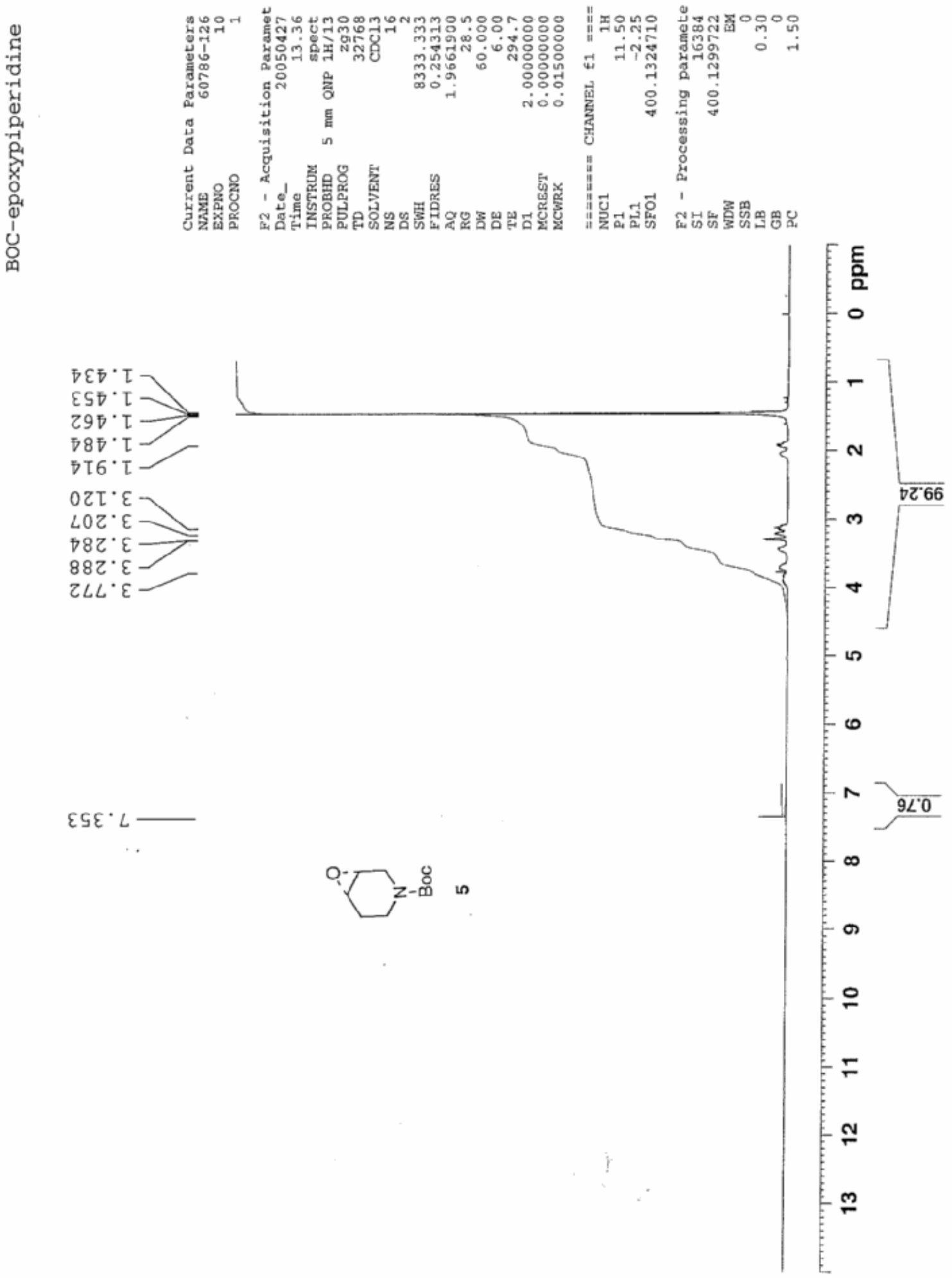



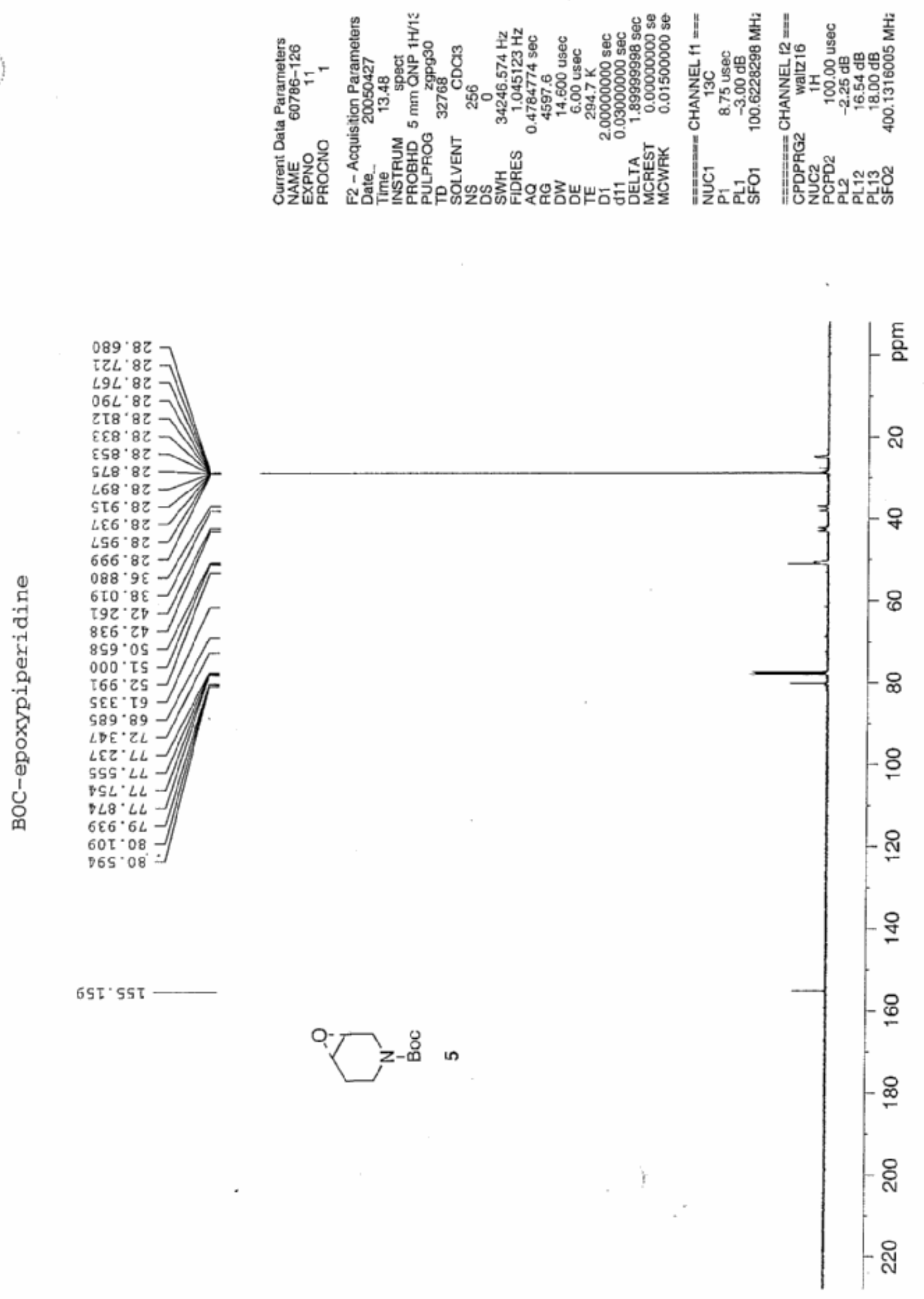


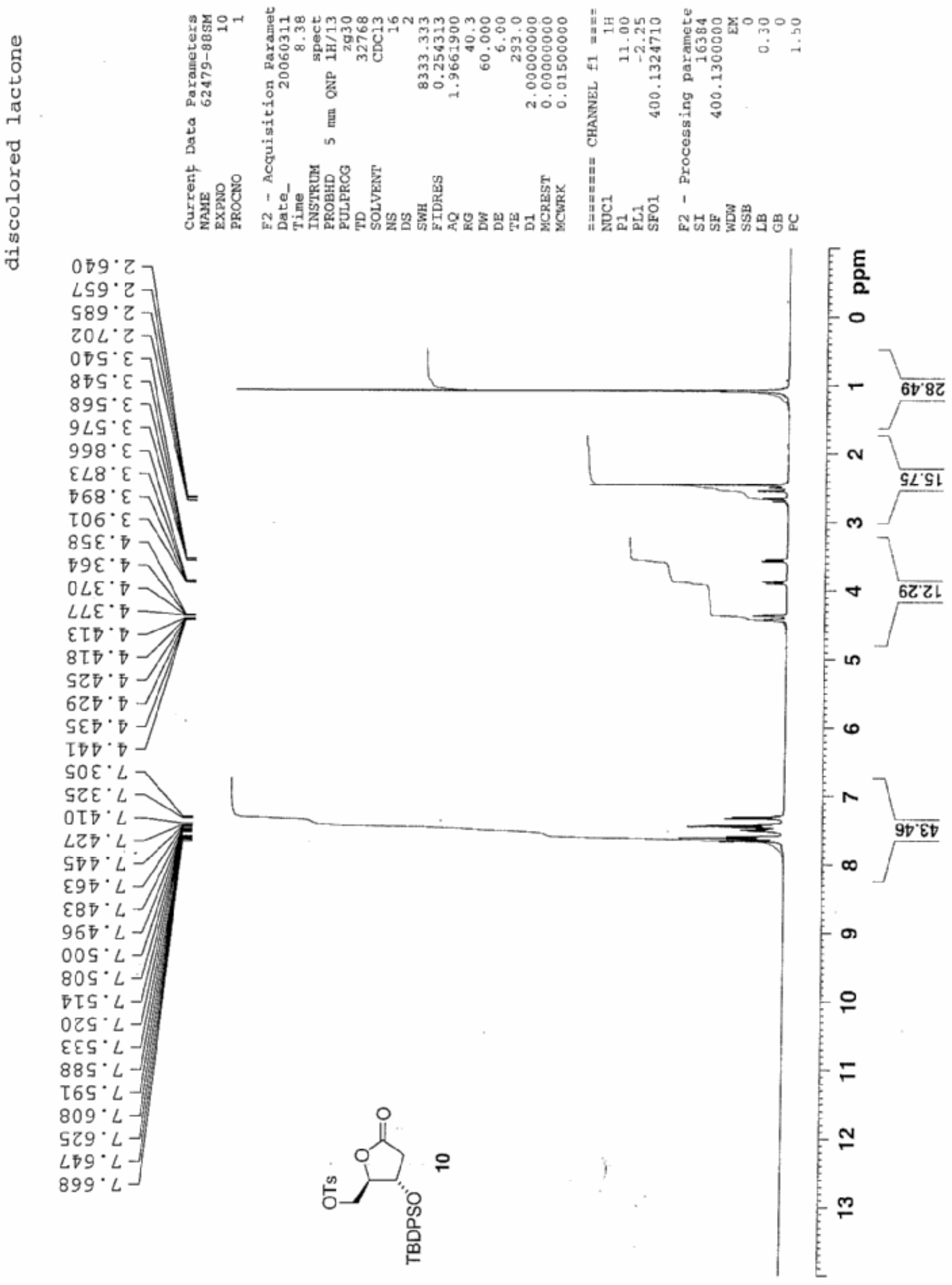



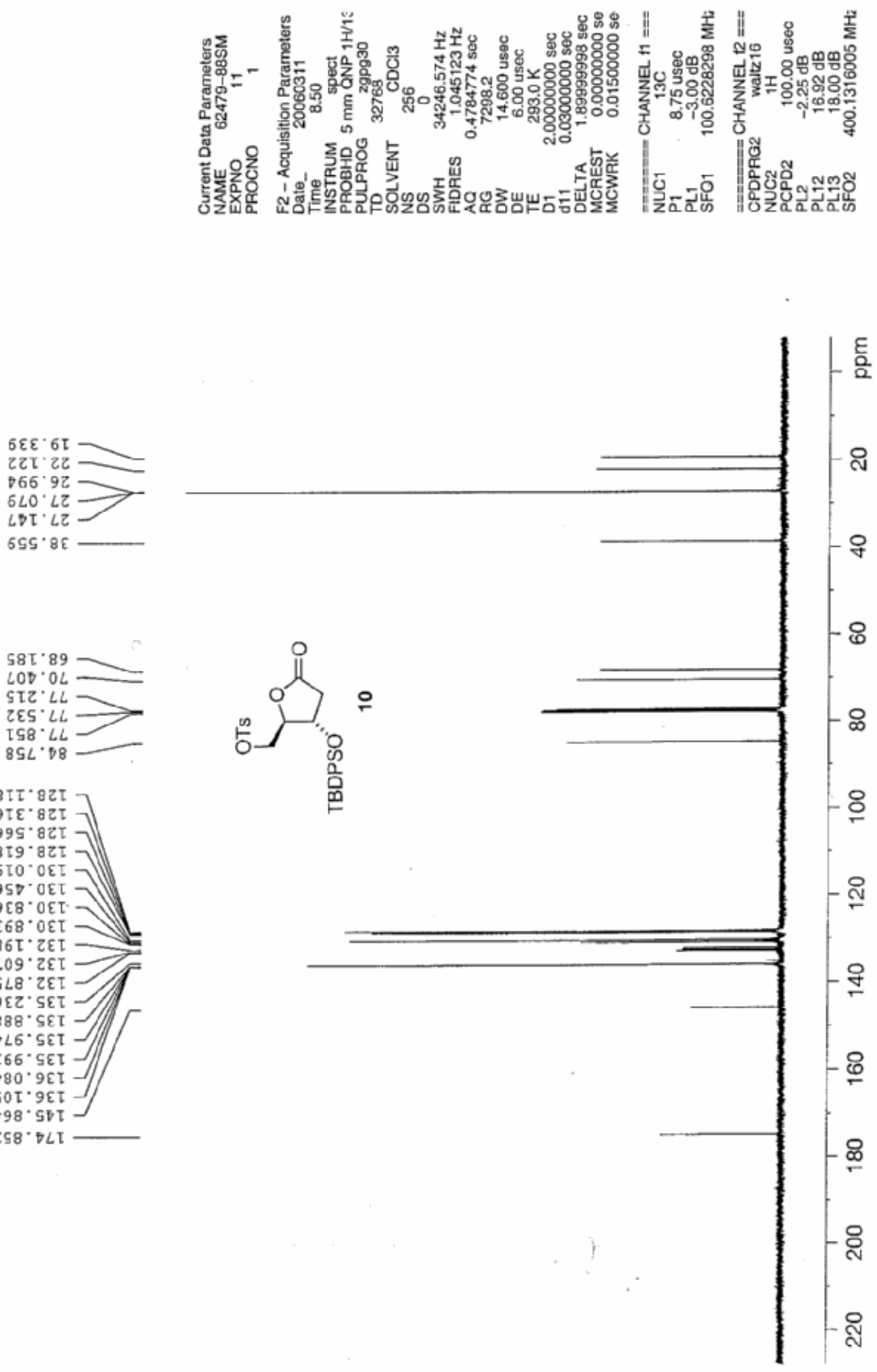


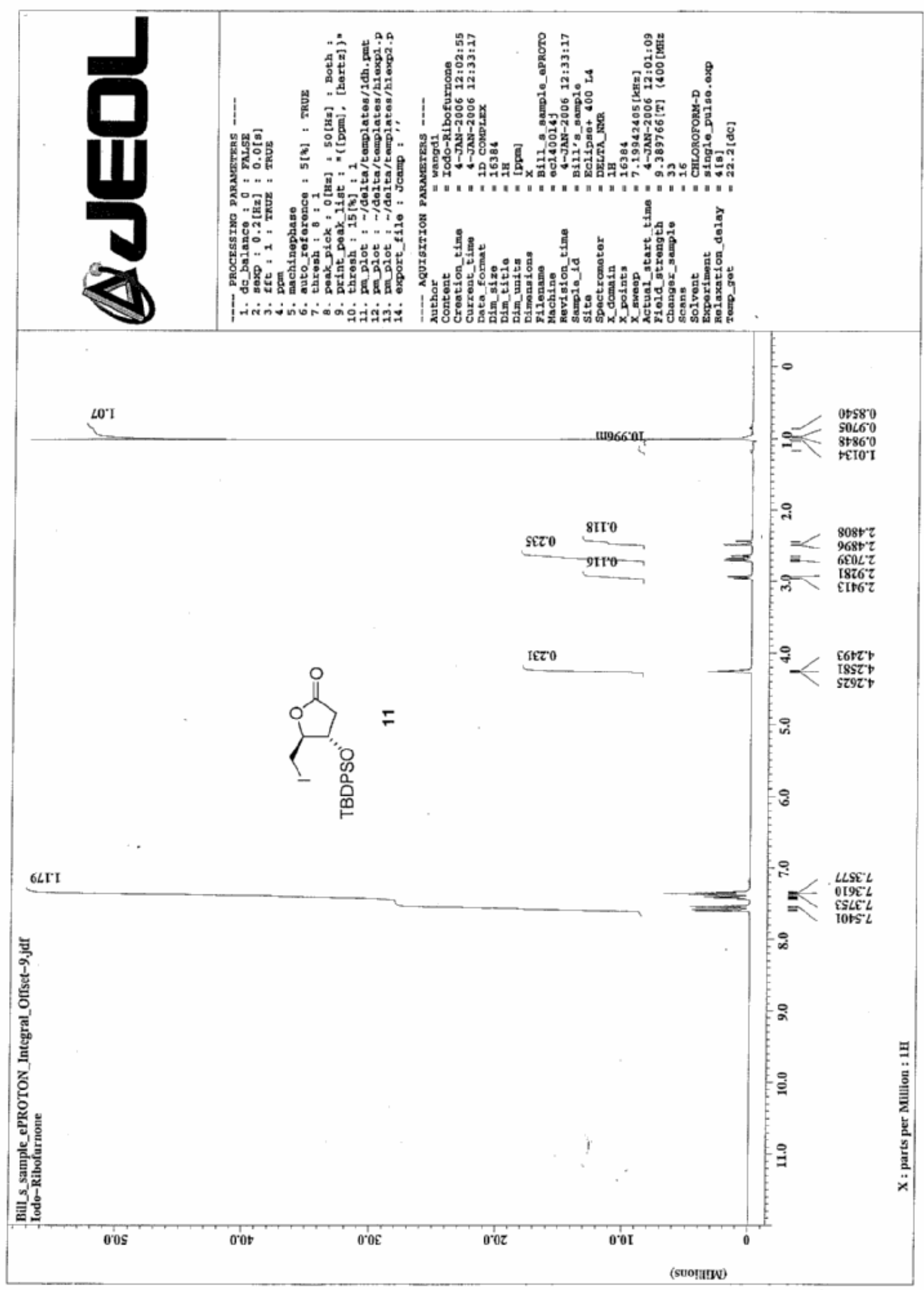




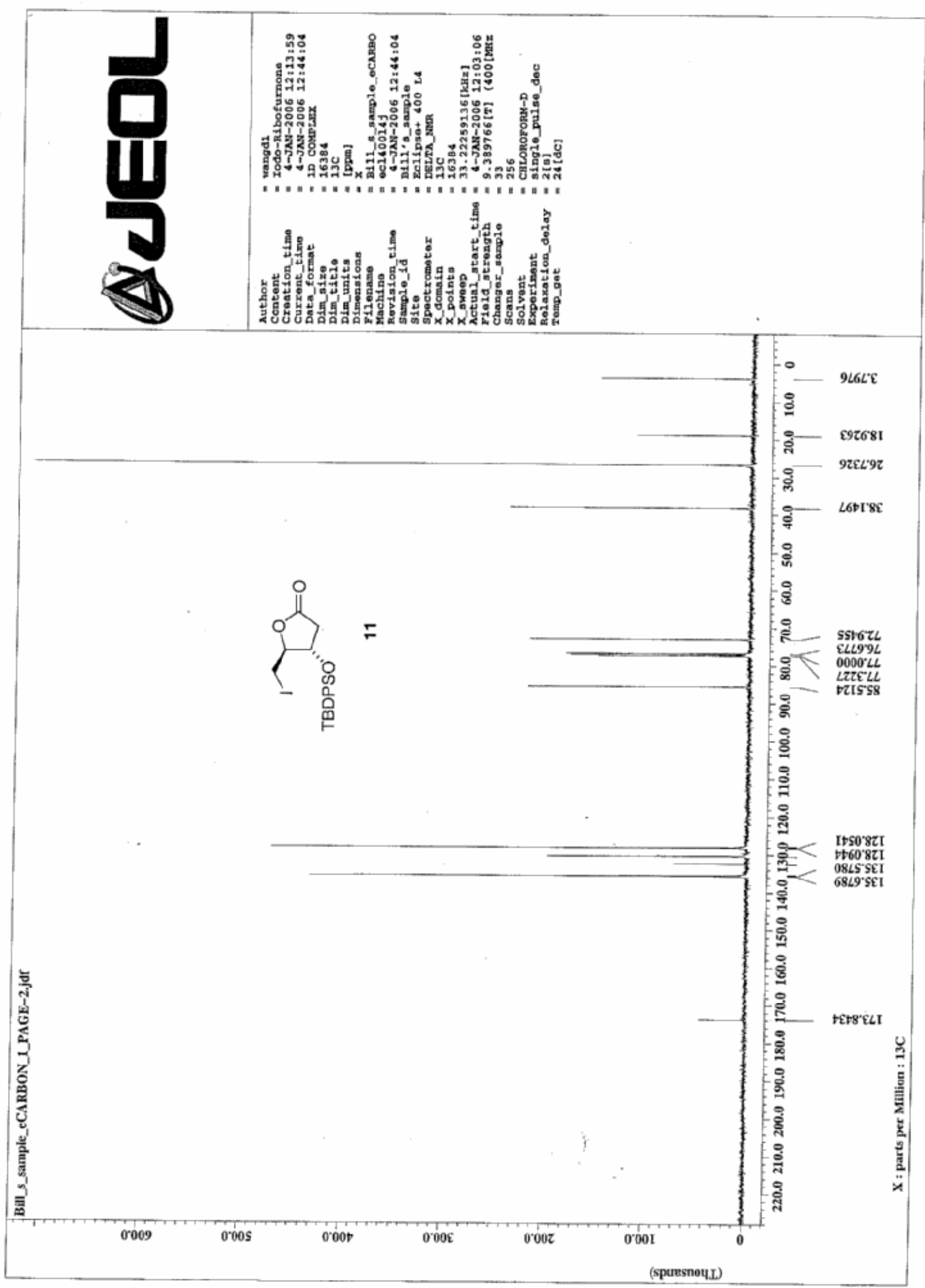




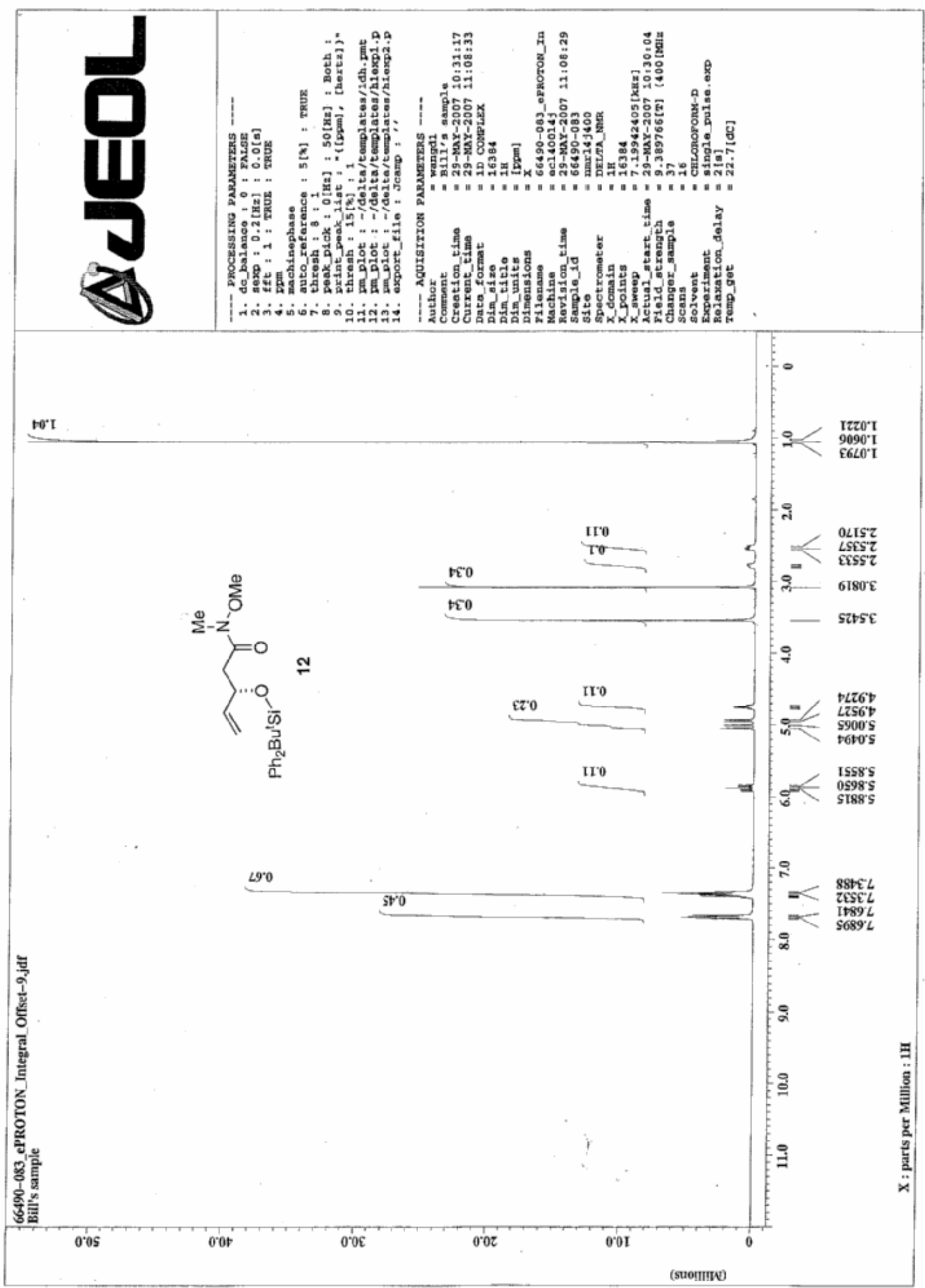




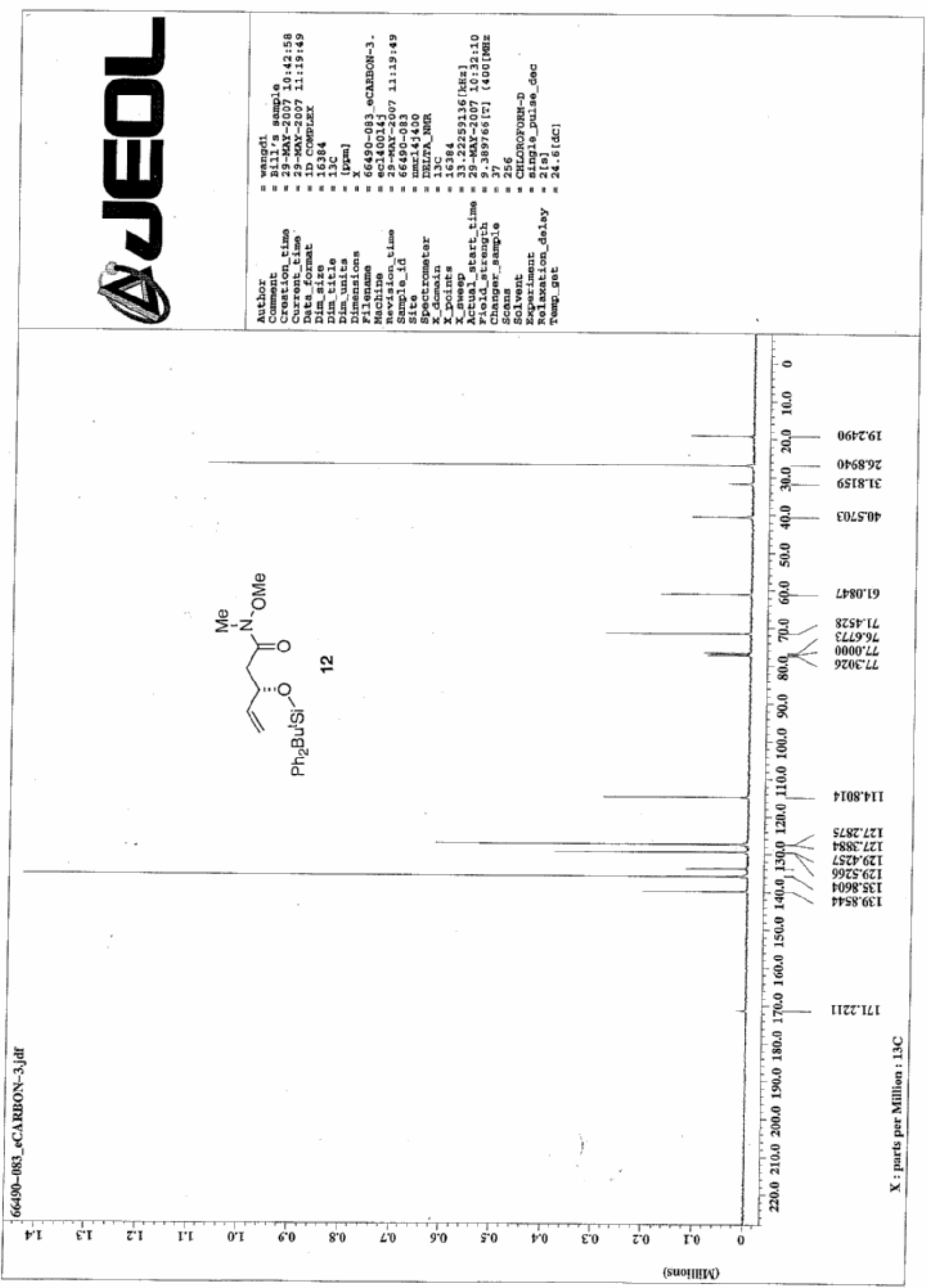




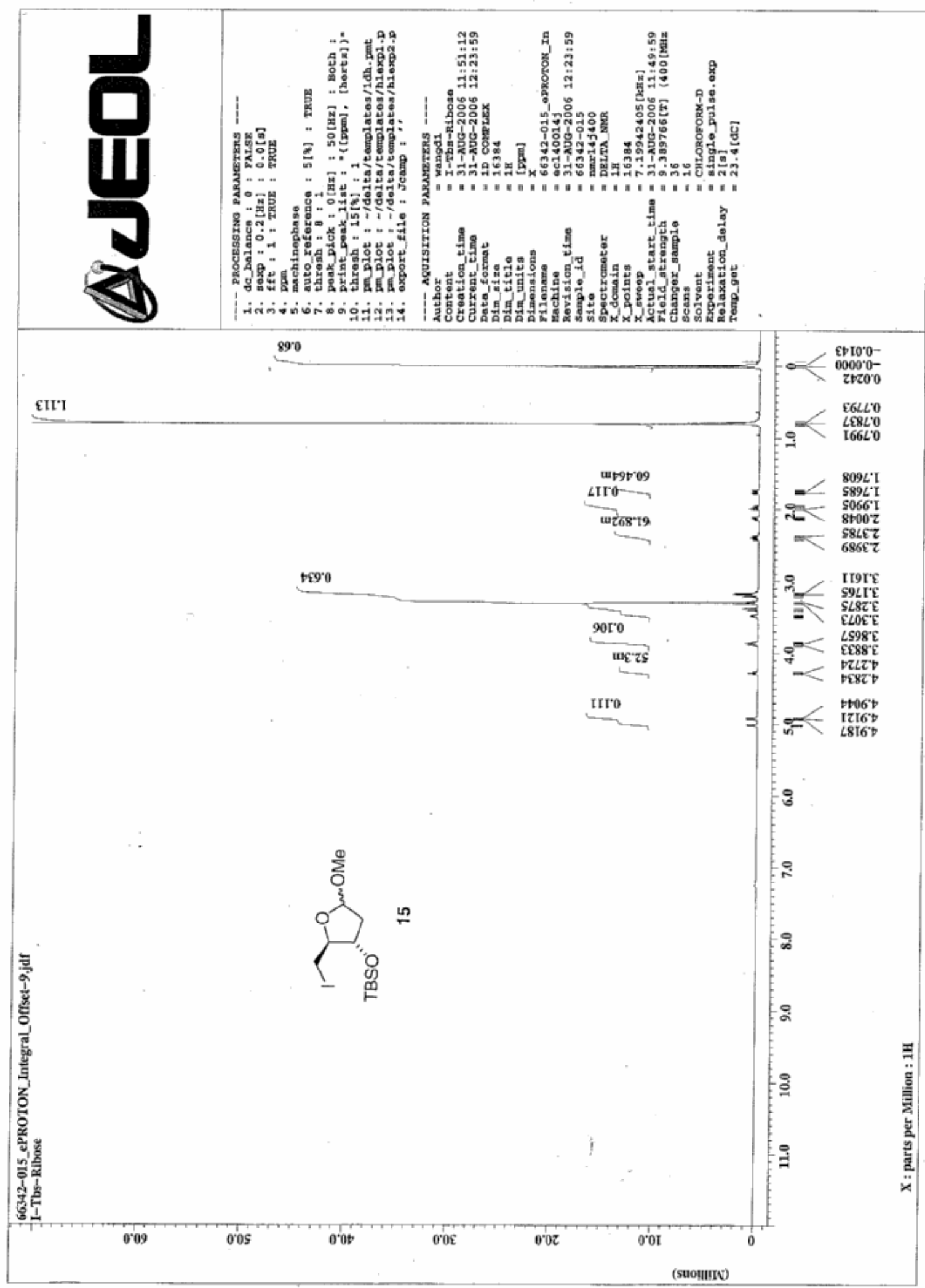




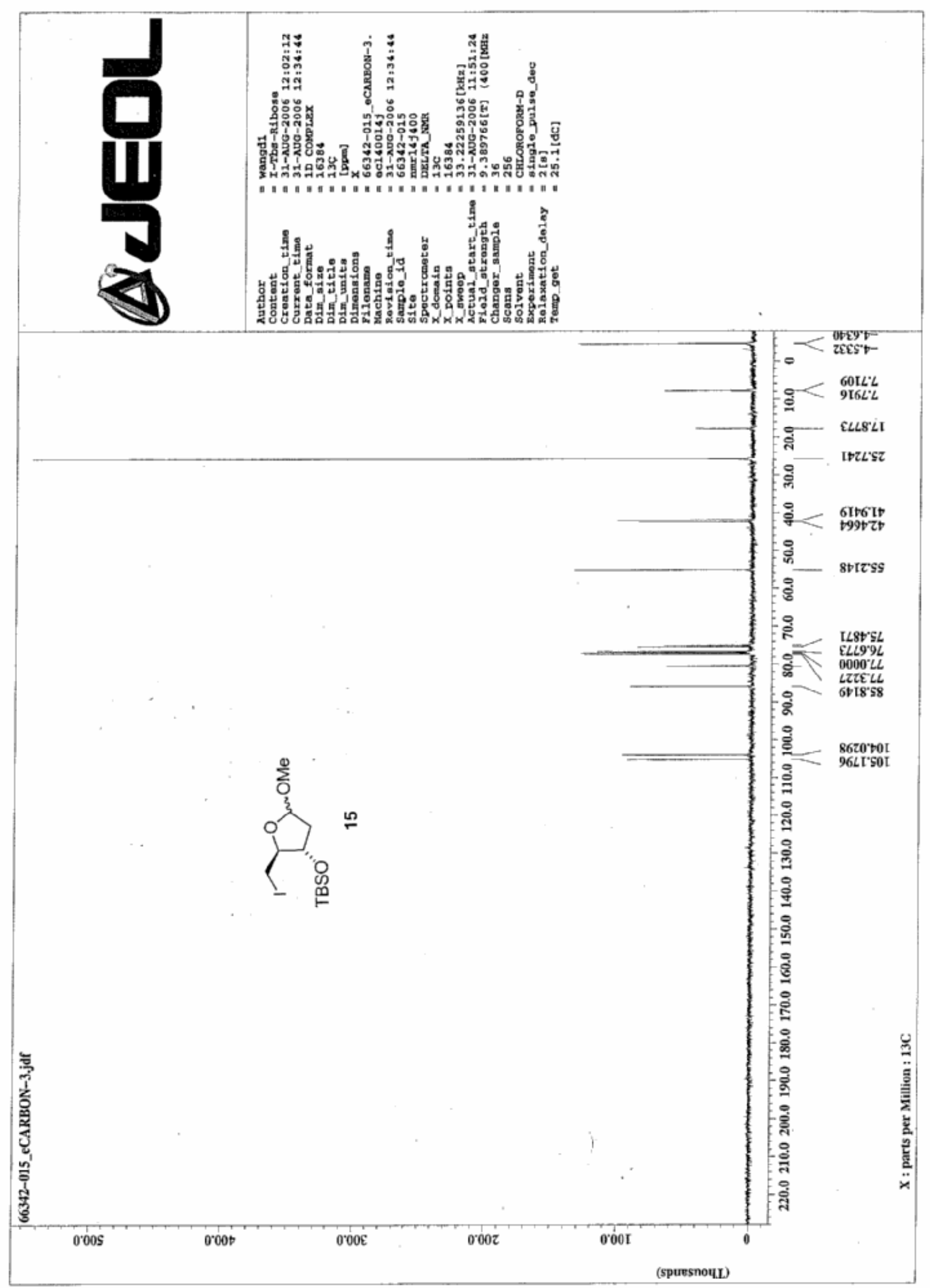




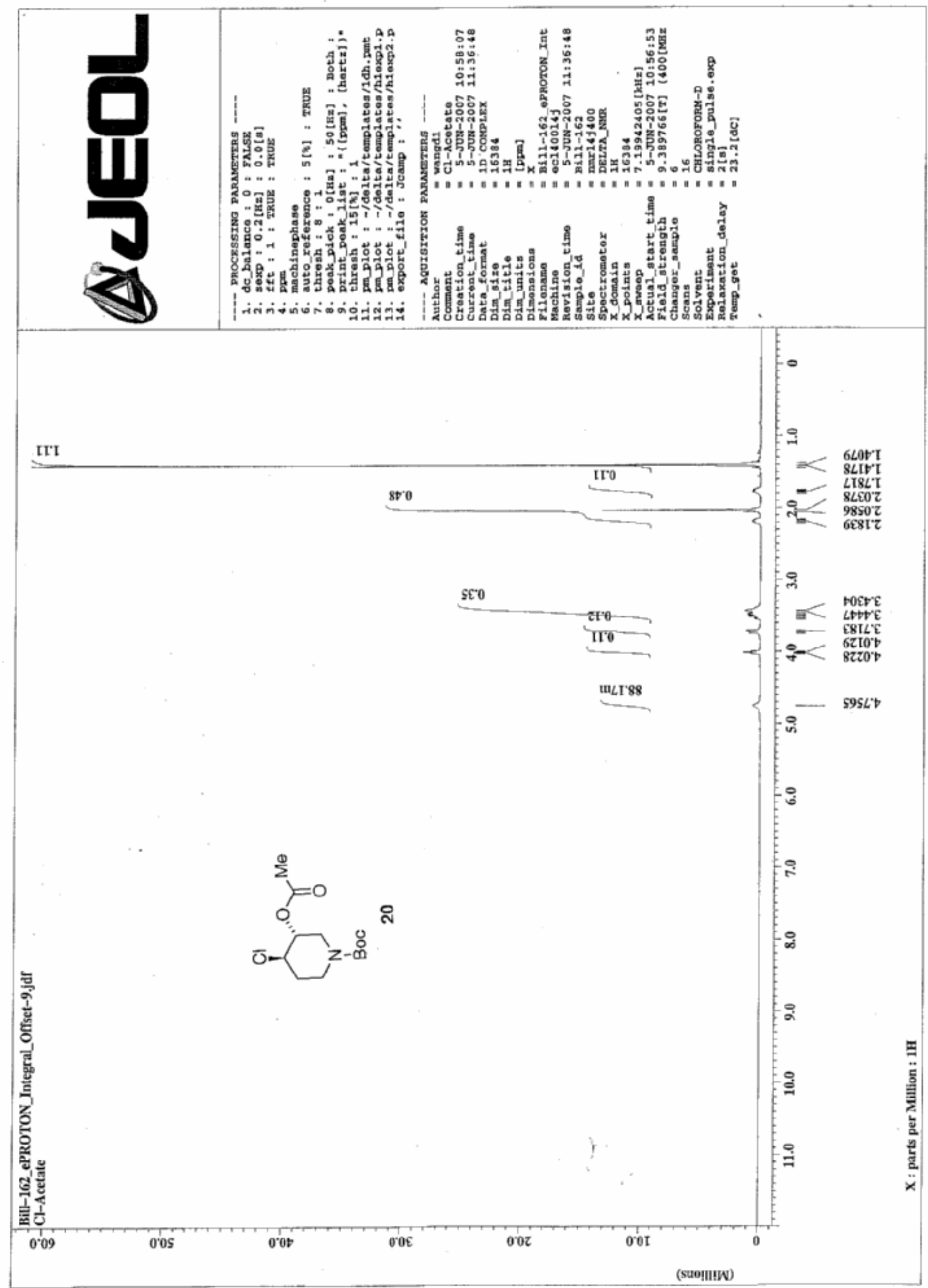




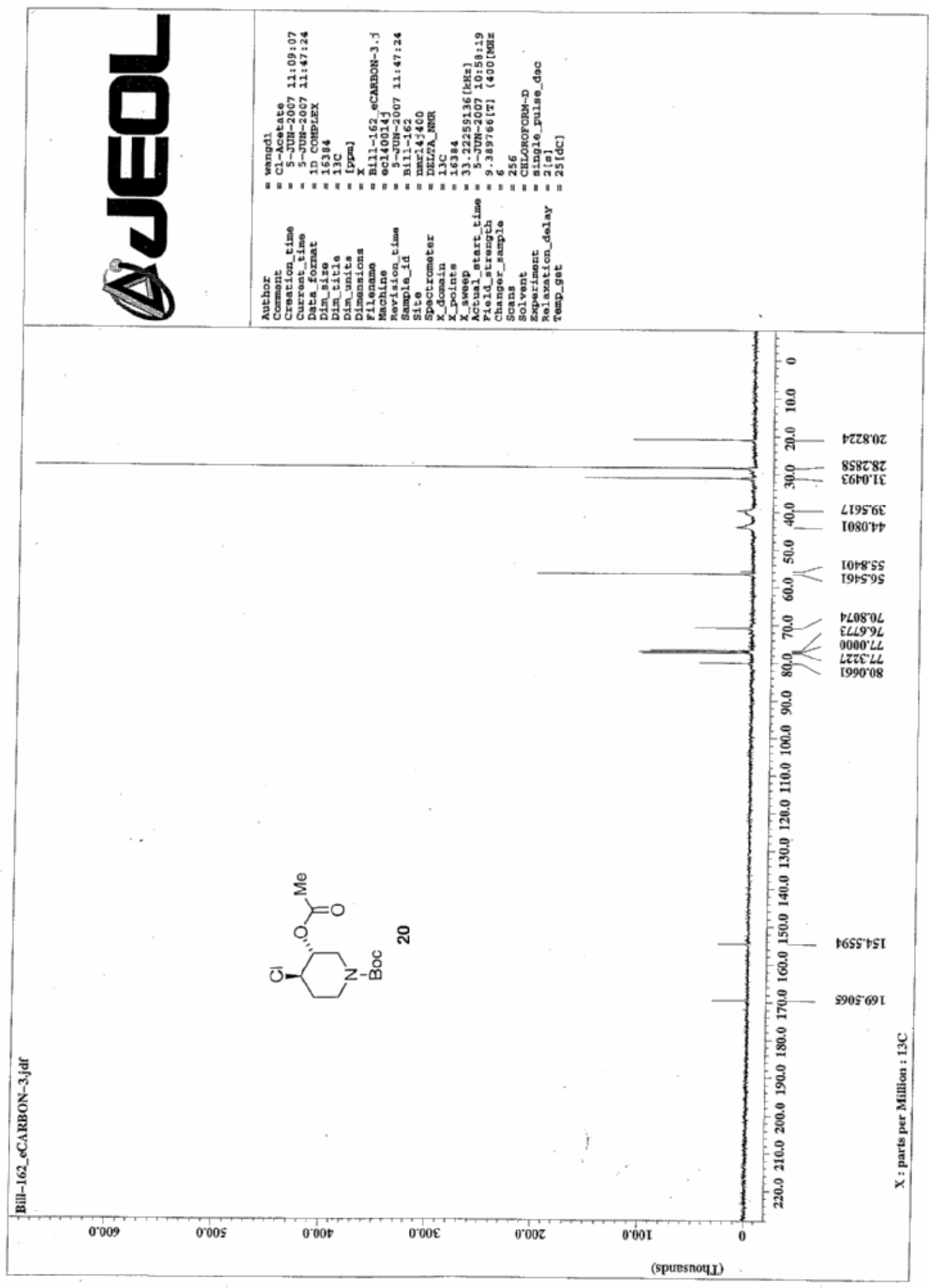




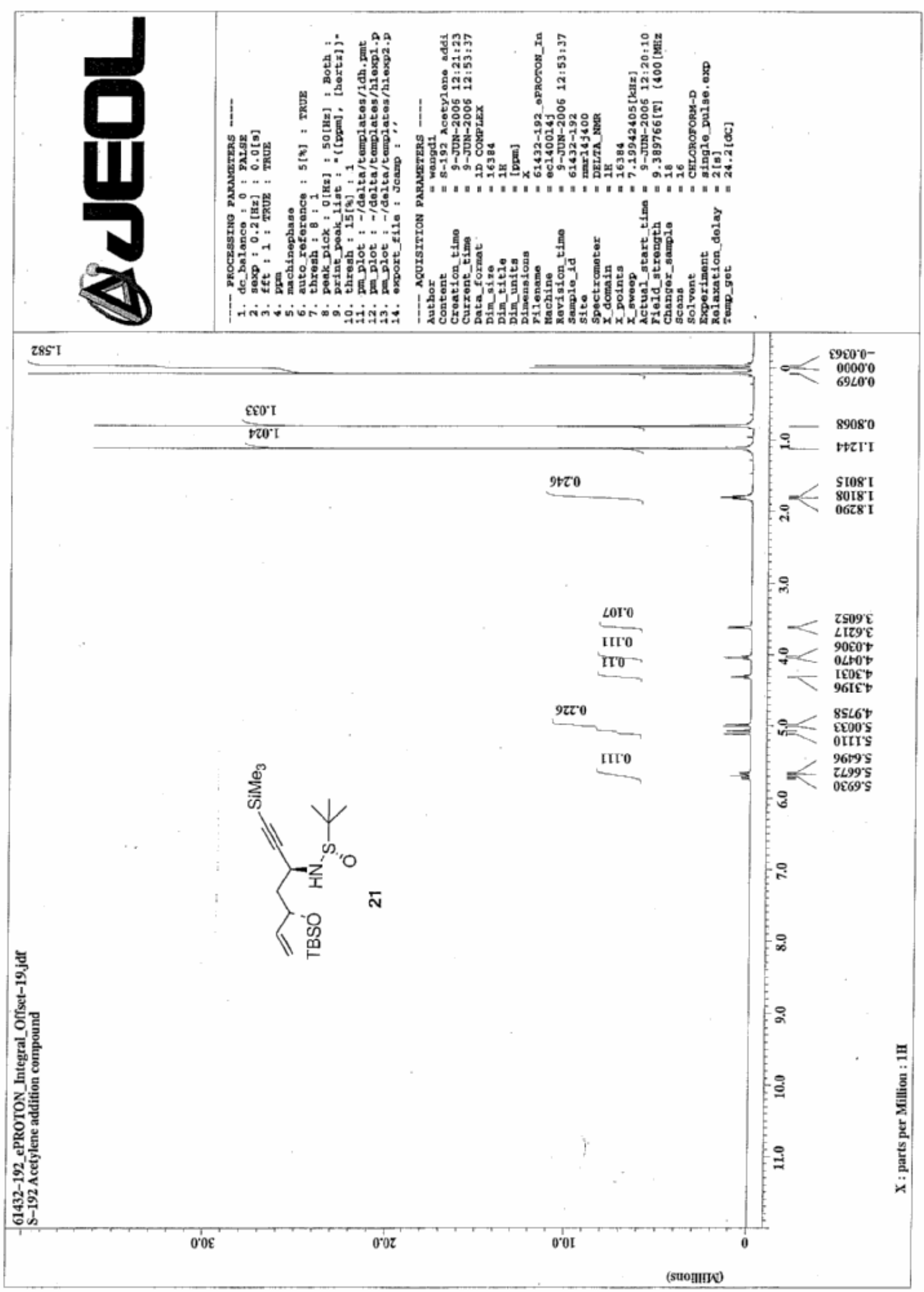


S-31

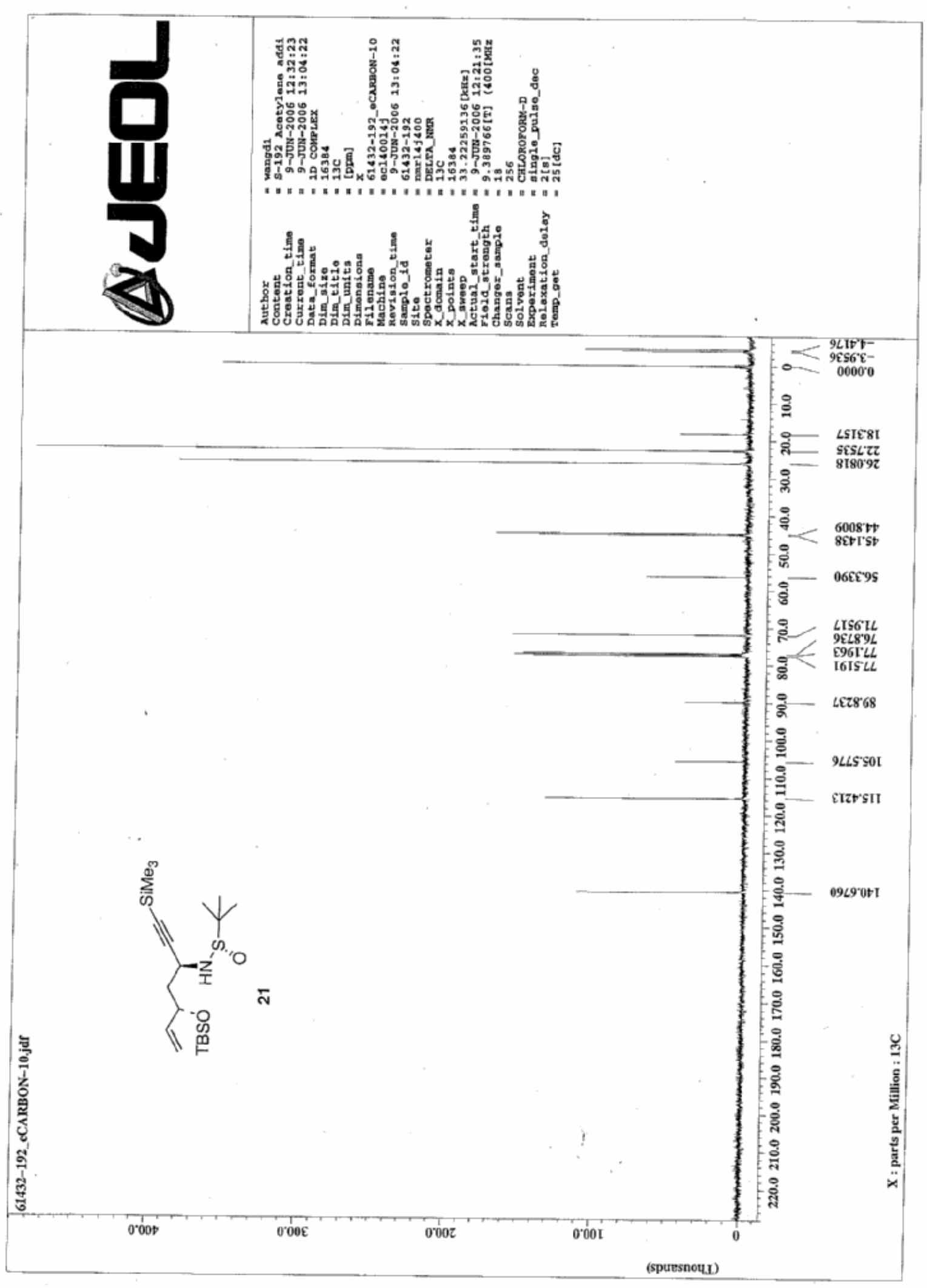




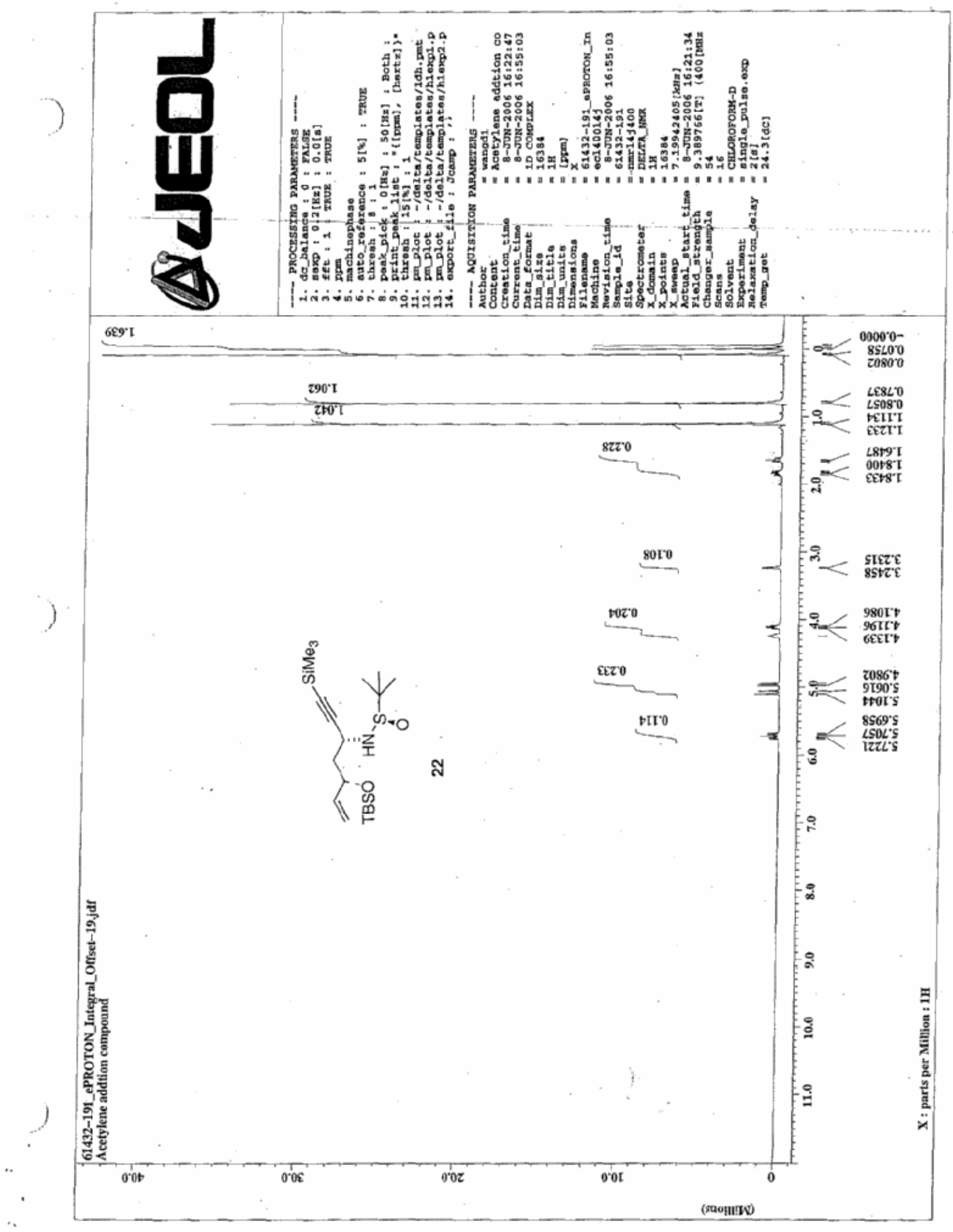


S-33

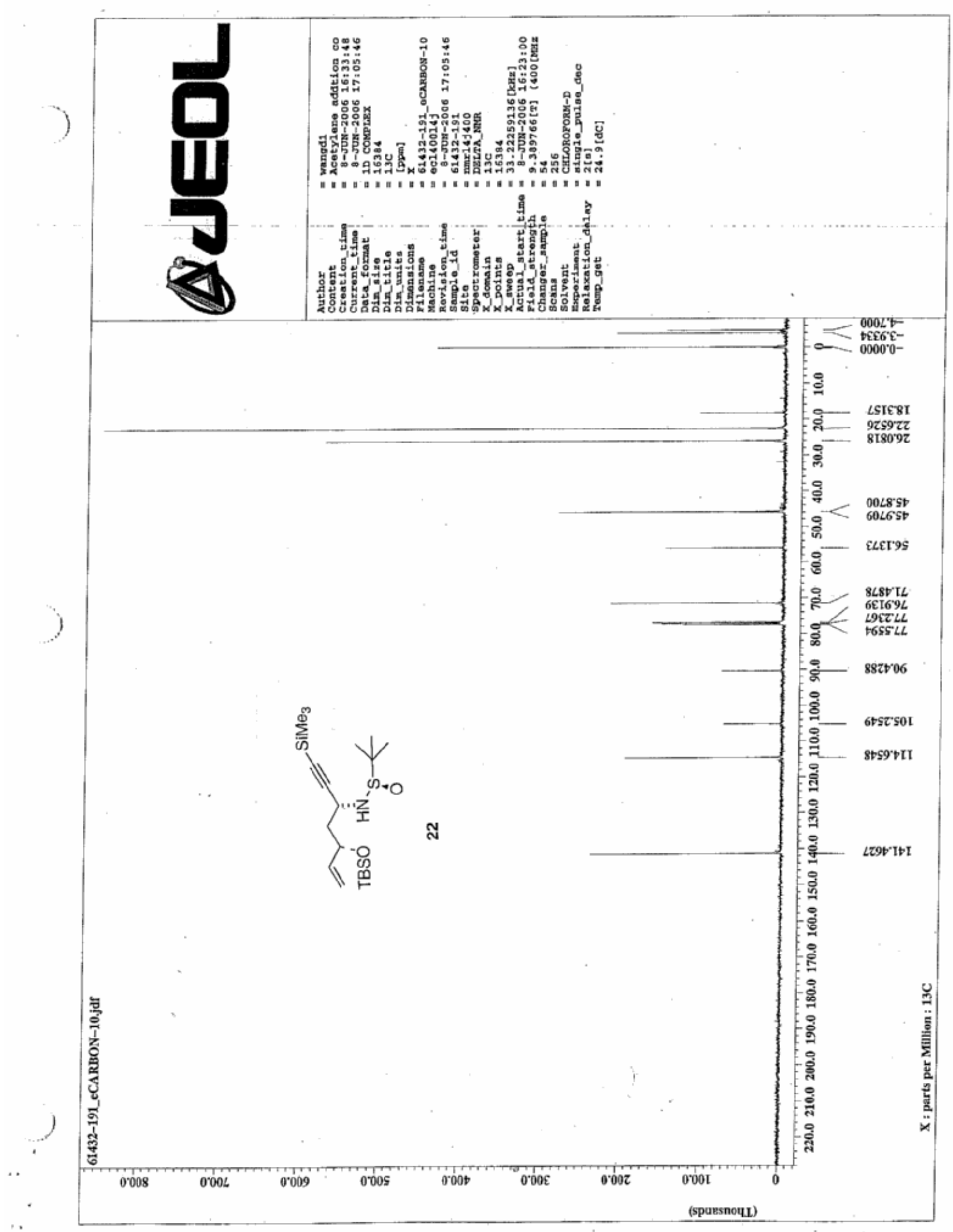

